\title{
COMBINING PARTICLE ACCELERATION AND CORONAL HEATING VIA DATA-CONSTRAINED CALCULATIONS OF NANOFLARES IN CORONAL LOOPS
}

\author{
C. Gontikakis ${ }^{1}$, S. Patsourakos ${ }^{2}$, C. Efthymiopoulos ${ }^{1}$, A. Anastasiadis ${ }^{3}$, and M. K. Georgoulis ${ }^{1}$ \\ ${ }^{1}$ Research Center for Astronomy and Applied Mathematics, Academy of Athens, Soranou Efessiou 4, 11528 Athens, Greece; cgontik@academyofathens.gr \\ ${ }^{2}$ Section of Astro-Geophysics, Department of Physics, University of Ioannina, 45110 Ioannina, Greece \\ ${ }^{3}$ National Observatory of Athens, Institute for Astronomy, Astrophysics, Space Applications and Remote Sensing, GR-15236, Palaia Penteli, Greece \\ Received 2012 November 16; accepted 2013 May 16; published 2013 June 25
}

\begin{abstract}
We model nanoflare heating of extrapolated active-region coronal loops via the acceleration of electrons and protons in Harris-type current sheets. The kinetic energy of the accelerated particles is estimated using semianalytical and test-particle-tracing approaches. Vector magnetograms and photospheric Doppler velocity maps of NOAA active region 09114, recorded by the Imaging Vector Magnetograph, were used for this analysis. A current-free field extrapolation of the active-region corona was first constructed. The corresponding Poynting fluxes at the footpoints of 5000 extrapolated coronal loops were then calculated. Assuming that reconnecting current sheets develop along these loops, we utilized previous results to estimate the kinetic energy gain of the accelerated particles. We related this energy to nanoflare heating and macroscopic loop characteristics. Kinetic energies of $0.1-8 \mathrm{keV}$ (for electrons) and $0.3-470 \mathrm{keV}$ (for protons) were found to cause heating rates ranging from $10^{-6}$ to $1 \mathrm{erg} \mathrm{s}^{-1} \mathrm{~cm}^{-3}$. Hydrodynamic simulations show that such heating rates can sustain plasma in coronal conditions inside the loops and generate plasma thermal distributions that are consistent with active-region observations. We concluded the analysis by computing the form of X-ray spectra generated by the accelerated electrons using the thick-target approach. These spectra were found to be in agreement with observed X-ray spectra, thus supporting the plausibility of our nanoflare-heating scenario.
\end{abstract}

Key words: Sun: activity - Sun: corona - Sun: flares - Sun: magnetic topology

Online-only material: color figure

\section{INTRODUCTION}

A strong candidate mechanism to explain coronal heating is the formation of small-scale, still-undetected reconnection events called nanoflares (Parker 1988). Reconnection events are sites where coronal magnetic energy is transformed into energy of accelerated particles, eventually producing heating. Solar flares constitute a well-documented case where accelerated particles, through reconnection, attain higher and higher kinetic energies, thus raising the plasma temperature while creating at the same time particle beams of supra-thermal kinetic energies (Birn \& Priest 2007).

Particle acceleration and the thermodynamic response of the plasma to heating are phenomena that were typically studied in isolation. In fact, a unifying study of particle acceleration, direct coronal heating, and the thermodynamic response of the observed plasma structures such as coronal loops seems hardly tractable, given the wide range of spatial and/or temporal scales involved. Indeed, these scales range from the dissipation scale (i.e., the scale of the current sheet thickness, which is of the order of centimeters or meters), to the scale of macroscopic phenomena taking place in the observed coronal structures (on the order of several tens to $\simeq 100 \mathrm{Mm}$; Klimchuk 2006).

In the present work, we report on the results of an attempt to connect direct coronal heating, and its thermodynamic response, to particle acceleration. To this end, we use several simplifying assumptions in the theoretical and numerical treatment of each individual process considered. Furthermore, we empirically constrain our assumptions by exploiting available observational information such as measurements and estimates of the magnetic and the velocity field vectors in a particular active region (AR).

As a starting point, we adopt Parker's hypothesis (Parker 1972) that plasma motions at the photospheric level stress, twist, and entangle the coronal magnetic field lines. This process converts the plasma kinetic energy at the footpoints of coronal loops to non-potential (free) magnetic energy stored in the coronal magnetic fields. According to Parker (1988), when the magnetic field stress reaches a critical point, the stored free magnetic energy should be released to the plasma via reconnection events. For quantitative calculations, one equates the work rate done by the photospheric motions to the observed radiated energy in the corona. One thus finds an estimate of the mean critical inclination of flux tubes with respect to the direction normal to the solar surface at which a reconnection event should take place. The resulting inclination angle, called the Parker angle, is derived through numerical simulations or analytical estimations. For ARs, the Parker angle takes values in the range of $5^{\circ}-20^{\circ}$, depending on the applied physical mechanism (Galsgaard \& Nordlund 1996; Klimchuk 2006; Rappazzo et al. 2007). The reason why the Parker angle appears preferentially in the above range (instead of taking values below $1^{\circ}$ or around $90^{\circ}$ ) has been interpreted in some numerical simulations as an effect of the temporal evolution of a reconnecting current sheet undergoing tearing instability (Dahlburg et al. 2005).

Numerical simulations can also help us to understand how coronal magnetic fields are stretched and twisted due to photospheric plasma motions. These magnetic fields develop complex, unstable current sheets (see, for example, Galsgaard \& Nordlund 1996). In some cases, it was found that there is a statistical equilibrium established between the energy supplied at the loop footpoints and the energy released throughout the whole structure of coronal loops via current sheets (Galsgaard \& Nordlund 1996; Hendrix et al. 1996). The simulated current sheets are of various types, scales, and forms. In particular, they vary from large monolithic structures extending over the loop's 
length (Galsgaard \& Nordlund 1996) to cascades of smaller structures exhibiting a range of different sizes and spatial distributions (Galsgaard \& Nordlund 1996; Hendrix et al. 1996; Rappazzo et al. 2007).

The above simulations show the dependence of the Parker angle on the loop parameters. However, they still present a high degree of idealization which may affect the computed Parker angle values. These include the simplified representation of the region between the photosphere and the corona (Klimchuk 2006), as well as the omission of the flux-tube divergence with height.

As our basic mechanism to explain the magnetic energy release during flares and nanoflares, we adopt the acceleration of particles inside reconnecting current sheets. A crucial parameter for achieving a viable simulation of this type is the "guide" magnetic field, i.e., a magnetic field component parallel to the electric field that accelerates the particles. The main effect of the guide magnetic field is to change the trajectories of the charged particles, thus enhancing motion parallel to the electric field (Litvinenko \& Somov 1993; Litvinenko 1996; Litvinenko 2000). In a previous work (Efthymiopoulos et al. 2005), these particles' motions were studied for the case of a Harris-type reconnecting current sheet model by means of a Hamiltonian formalism. The result was a general formula predicting the maximum kinetic-energy gain of accelerated particles as a function of the initial energy of the particles and the parameters of the current sheet (thickness, field strengths). This formula is used in the present work to estimate the kinetic energy of the accelerated particles in the extrapolated coronal loops. We also note that, as found in Gontikakis et al. (2007) and Anastasiadis et al. (2008), the kinetic-energy distributions of accelerated particles through single or multiple current sheets (Harris type or X-point) are subject to upper limits due to the existence of a maximum possible kinetic-energy gain of the particles.

Another input of our present analysis is estimates of the current sheet thicknesses in these reconnection events. For this, we exploit results of recent particle-in-cell studies, in which the magnetic reconnection in solar flares takes place in the presence of a guide magnetic field (Hesse et al. 1999; Cassak et al. 2008). One then finds that the thickness of the diffusive current sheet is of the order of the electron gyroradius.

Considering now the response of the coronal plasma to nanoflares, we rely on time-dependent hydrodynamic simulations (Patsourakos \& Klimchuk 2005; Patsourakos et al. 2004). In these models, the cumulative effect of a large number of nanoflares, releasing energy in a coronal loop, is simulated in a way that allows a direct comparison between simulations and observations from telescopes such as the Transition Region and Coronal Explorer (Handy et al. 1998) or the Atmospheric Imaging Assembly (Lemen et al. 2012) on board the Solar Dynamic Observatory.

The structure of the paper is as follows. In Section 2, we discuss the observational data used to compute the structure of the magnetic field in the AR corona. Section 3 presents our modeling of coronal loops, leading to a derivation of values for the field strengths and the footpoint velocities of the field lines forming the loops. Section 4 explains our main assumptions used to derive needed values of the current sheet parameters. Section 5 presents the particle acceleration results. These are used as an input to compute, in Section 6, the overall loop heating caused by particle acceleration. In Section 7 , we present a simulation of what the X-ray spectra of the accelerated electrons would look like under a thick-target model. Section 8 contains

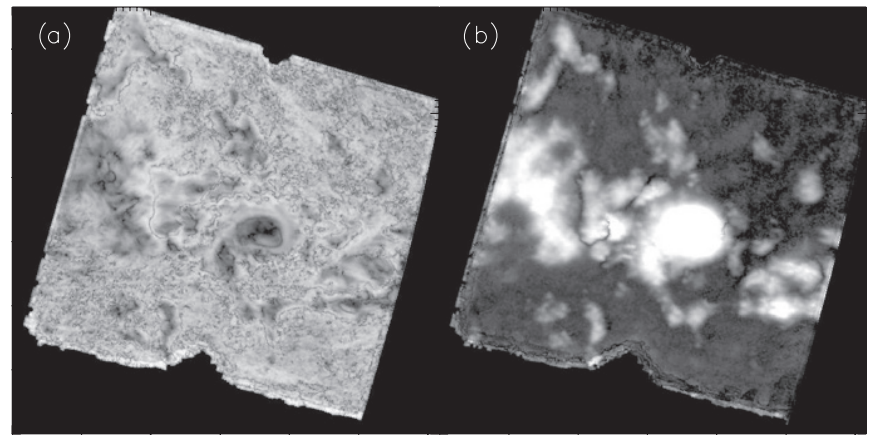

Figure 1. (a) Modulus of the velocity $v=\sqrt{v_{x}^{2}+v_{y}^{2}}$ computed with the MSR vector. The gray scale is set to range from 0.3 to $4.5 \mathrm{~km} \mathrm{~s}^{-1}$. (b) Modulus of the magnetic field from the vector magnetogram. The gray scale is set to range from 10 to $500 \mathrm{G}$.

comprehensive results on loop heating via nanoflares, as well as on the thermodynamic response of the plasma in the modeled coronal loops. Section 9 offers a discussion of our results and the limitations and validity of our modeling. Finally, Section 10 summarizes the basic conclusions of the study.

\section{OBSERVATIONS AND DATA TREATMENT}

The Imaging Vector Magnetograph (IVM) of the University of Hawaii's Mees Solar Observatory recorded a time series of 12 vector magnetograms of the AR 09114 spanning a $\simeq 4.5 \mathrm{hr}$ period on 2000 August 8 with a cadence of 20 minutes. These magnetograms have a field of view of $280^{\prime \prime} \times 280^{\prime \prime}$ and a spatial resolution of 0'.55 (Mickey et al. 1996). The IVM recorded the Stokes vector of the Fe I $6302.5 \AA$ photospheric spectral line.

Starting from the above data, we first resolve the azimuthal $180^{\circ}$ ambiguity in each magnetogram using the non-potential magnetic field calculation method of Georgoulis (2005), as refined in Metcalf et al. (2006). Then, we compute a mean vector magnetogram as well as a map of the associated photospheric horizontal velocity, calculated by means of the minimum structure reconstruction (MSR) technique (Georgoulis \& LaBonte 2006; see Figure 1).

Expressing the photospheric magnetic field vector in the local heliographic reference system, we can now perform a currentfree (potential) field extrapolation using the method proposed by Alissandrakis (1981). This computation was done in a cube up to an altitude of $70^{\prime \prime}$, or $\simeq 50 \mathrm{Mm}$, while the potential field extrapolation was performed on a $2 \times 2$ binned magnetogram.

\section{CORONAL LOOP MODELING}

Using the extrapolated potential fields, we now define the coronal loops and several associated quantities. We proceed via the following steps.

1. Coronal loop identification. We selected about 5000 extrapolated magnetic field lines all closing within the photospheric field of view. These magnetic field lines are identified as closed coronal loops. The loops' lengths (denoted by $L$ hereafter) range from 8 to $180 \mathrm{Mm}$. The loops' maximum heights range from $2.5 \mathrm{Mm}$ to $50 \mathrm{Mm}$.

2. Magnetic field strength. We computed a mean value $\bar{B}$ of the magnetic field along each coronal loop as in Mandrini et al. (2000) and Gontikakis et al. (2008). The mean magnetic field is found to statistically decrease with increasing loop length, with short loops $(L<20 \mathrm{Mm})$ yielding an average $\bar{B} \simeq 180 \mathrm{G}$ while long loops $(L>100 \mathrm{Mm})$ yield an 

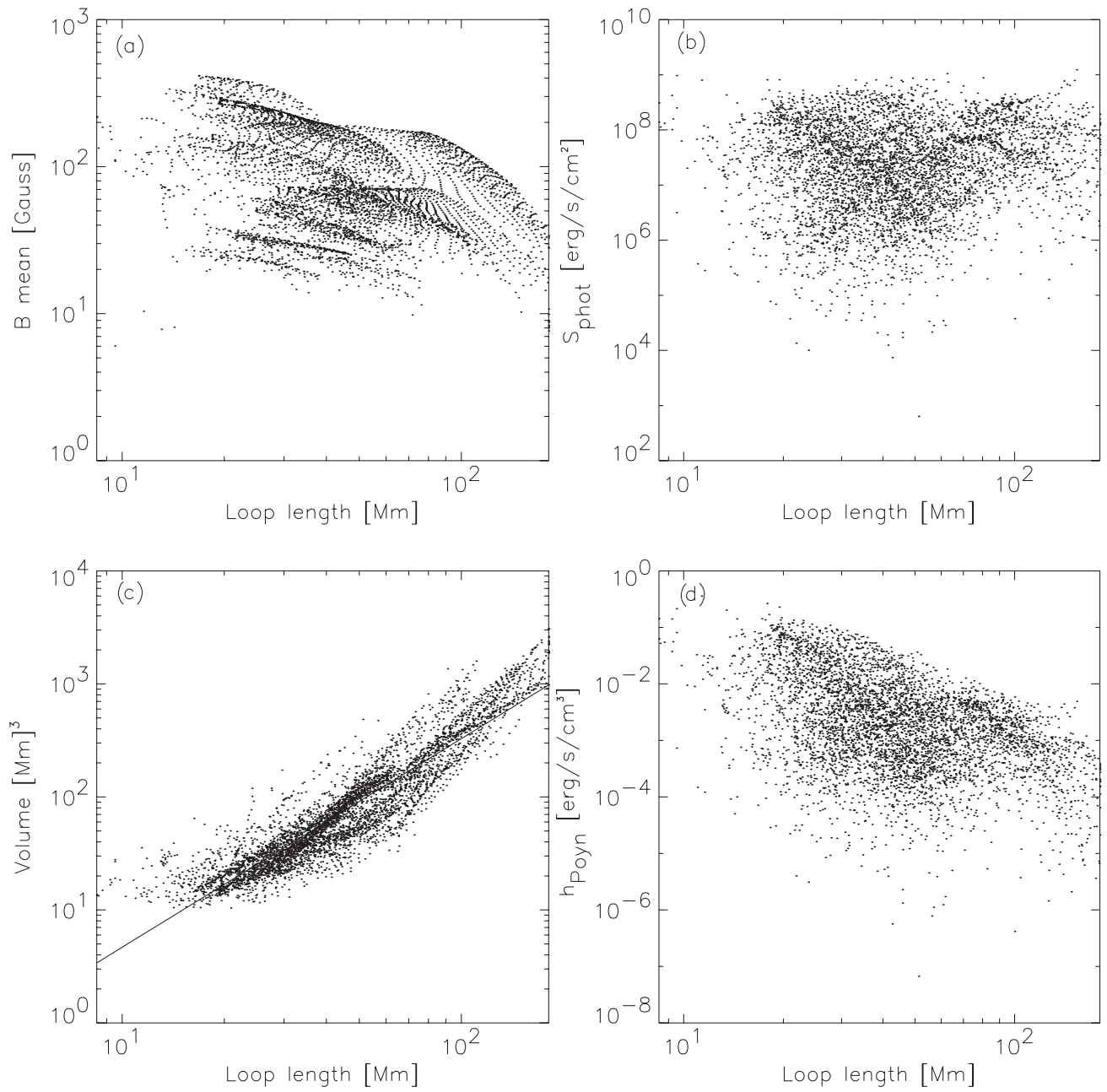

Figure 2. Scatter plots for several loop parameters as a function of loop length. These are (a) the mean field strength, (b) the Poynting flux at both footpoints of each loop, (c) the loop volume, and (d) the volumetric heating rate due to the Poynting flux. In panel (c), a fit is also shown.

average $\bar{B} \simeq 60 \mathrm{G}$. Figure 2(a) shows the mean magnetic field strength for each loop as a function of the loop's length.

3. Electric field strength. For each coronal loop, we calculate the photospheric electric field values at both footpoints $\boldsymbol{E}_{\text {phot }}=-(1 / c) \boldsymbol{v}_{\text {phot }} \times \boldsymbol{B}$ or

$$
\boldsymbol{E}_{\text {phot }}=\frac{1}{c}\left(v_{y} B_{z} \boldsymbol{x}-v_{x} B_{z} \boldsymbol{y}\right) .
$$

In Equation (1), $v_{x}$ and $v_{y}$ are the calculated horizontal components of the photospheric velocities, $B_{z}$ is the perpendicular magnetic field component, and $\boldsymbol{x}$ and $\boldsymbol{y}$ are the corresponding unit vectors. Equation (1) does not include $v_{z}$ because, in the MSR method, the vertical (cross-field) velocity component is assumed to be negligible.

4. Poynting flux through loops and supply of free magnetic energy. As an immediate consequence of step (3) above, we can deduce values of the Poynting flux normal to the solar surface at the loops footpoints. These values are expressed as

$$
S_{\text {foot }}=\frac{c}{4 \pi}\left(E_{x} B_{y}-E_{y} B_{x}\right) z,
$$

where $E_{x}=(1 / c) v_{y} B_{z}$ and $E_{y}=-(1 / c) v_{x} B_{z}$ are the photospheric electric field components. For each loop, two Poynting fluxes $\boldsymbol{S}_{\text {foot1 }}$ and $\boldsymbol{S}_{\text {foot2 }}$ are computed at the positions of the respective footpoints ( 1 or 2$)$. We should emphasize that while we have the information about the direction of the velocity vectors at the points of the observational grid, the local direction of smallscale motions at the footpoints of each coronal loop is inaccessible. For this reason, we ignore signs indicating an in or out Poynting flux through the loop and define, instead, the absolute sum $S_{\text {phot }}=\left|S_{\text {foot1 }}+S_{\text {foot2 }}\right|$ as a rough measure of the Poynting flux supplied to the loop due to photospheric motions. For a single, isolated loop, the supply of free magnetic energy should be considered a result of the relative plasma motions at both footpoints, since both distort the magnetic field. In fact, this distortion results in currents inside the loops, whose magnetic field now becomes non-potential. Furthermore, Equation (2) is not exact as it does not precisely resolve the relative motions at the footpoints. However, it arguably gives the correct order of magnitude for the Poynting flux. Finally, Equation (2) neglects the changes of the magnetic field due to the emergence or submergence of magnetic flux (Harra et al. 2012). This is justified in the case of AR 09114, since this is a fully developed and non-decaying AR at the time of the observations.

The calculated $S_{\text {phot }}$ (Figure 2(b)) has an average of $7 \times$ $10^{7} \mathrm{erg} \mathrm{s}^{-1} \mathrm{~cm}^{-2}$ and a standard deviation of $\simeq 10^{8} \mathrm{erg} \mathrm{s}^{-1} \mathrm{~cm}^{-2}$. Ninety-five percent of its calculated values are in the range 
$5 \times 10^{5}-5 \times 10^{8} \mathrm{erg} \mathrm{s}^{-1} \mathrm{~cm}^{-2}$, regardless of the loop length. In Figure $2(d)$, we show the loop heating $h_{\text {Poynt }}$ that would be produced if all the magnetic field energy input corresponding to the Poynting flux $S_{\text {phot }}$ was converted to thermal energy. This quantity is computed for comparison with the heating terms derived using particle acceleration and presented in Section 6. As $h_{\text {Poynt }}$ is an average value over time and space, it does not simulate the intermittent nature of nanoflares. It is given as

$$
h_{\text {Poynt }}=\frac{A_{\text {phot }}\left(\left|S_{\text {foot1 }}+S_{\text {foot2 }}\right|\right)}{V_{\text {loop }}} .
$$

In Equation (3), $A_{\text {phot }}$ is the cross section at the footpoints of each loop. These cross sections are equal to the square of the magnetogram's binned pixel size, i.e., $0.6 \mathrm{Mm}^{2}$. $V_{\text {loop }}$ is the loop volume (Figure 2(c)). Here, $V_{\text {loop }}$ is calculated by integrating the volume of infinitesimal cylinders corresponding to the different cross sections along the loop. We note that in subsequent calculations, the cross section is allowed to vary along each loop in order to conserve the magnetic flux passing through it. The constant magnetic flux of each loop equals the average magnetic flux calculated at its two footpoints. In Figure 2(c), we performed a logarithmic fit that shows that the volume as a function of the loop length is described by $V_{\text {loop }}=0.07 L^{1.85}$. This expression is used in Section 6 to describe a loop heating function.

In Figure 2(d), we see that $h_{\text {Poynt }}$ is in the range of $10^{-5}$ to $0.4 \mathrm{erg} \mathrm{s}^{-1} \mathrm{~cm}^{-3}$. Moreover, $h_{\text {Poynt }}$ is decreasing as a function of loop length $L$ due to the latter's inverse dependence on the loop

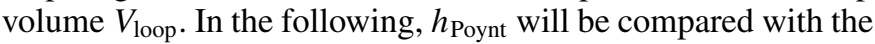
heating rates computed by the particles' acceleration.

We finally note that instead of the sum $\left|S_{\text {foot1 }}+S_{\text {foot2 }}\right|$, we have also made calculations using $\left|S_{\text {foot1 }}\right|+\left|S_{\text {foot2 }}\right|$ as an upper estimate of the total Poynting flux $S_{\text {phot }}$ through a loop. Both expressions lead to quite similar results. Hereafter, we will only refer to calculations performed using $\left|S_{\text {foot } 1}+S_{\text {foot } 2}\right|$.

\section{CURRENT SHEET MODELING}

We now proceed to model current sheets formed along our extrapolated coronal loops. While a reasonable assumption is that each loop should include several current sheets at subresolution scales, it will be shown below that the resulting coronal heating by the cumulative result of multiple current sheets distributed inside the loop is equivalent to the heating from a single "average" current sheet extending from footpoint to footpoint and consisting of a tangential discontinuity with a variable magnetic field vector across its surface. Figure 3 presents a schematic view of a stretched-out cylindroidal coronal loop that contains one such current sheet. Since the magnetic flux is conserved along each loop, the loop's cross section increases as we move from one of the footpoints toward the loop's apex. The current sheet is on the reconnection plane (dashed cut), which includes the cylindroid's axis. In Figure 3, the reconnection plane extends through the entire cylindroid. As shown in Section 5, this does not influence the resulting heating. The plane of the current sheet coincides with the plane of the discontinuity formed due to shearing motions at the photospheric level that presumably lead to the formation of two distinct magnetic-flux domains within the loop volume.

The magnetic field vectors in Figure 3 are depicted with different orientations above and below the reconnection plane. The angle $\theta_{D}$ formed between two adjacent flux tubes at the discontinuity is assumed to be equal to twice the Parker angle.

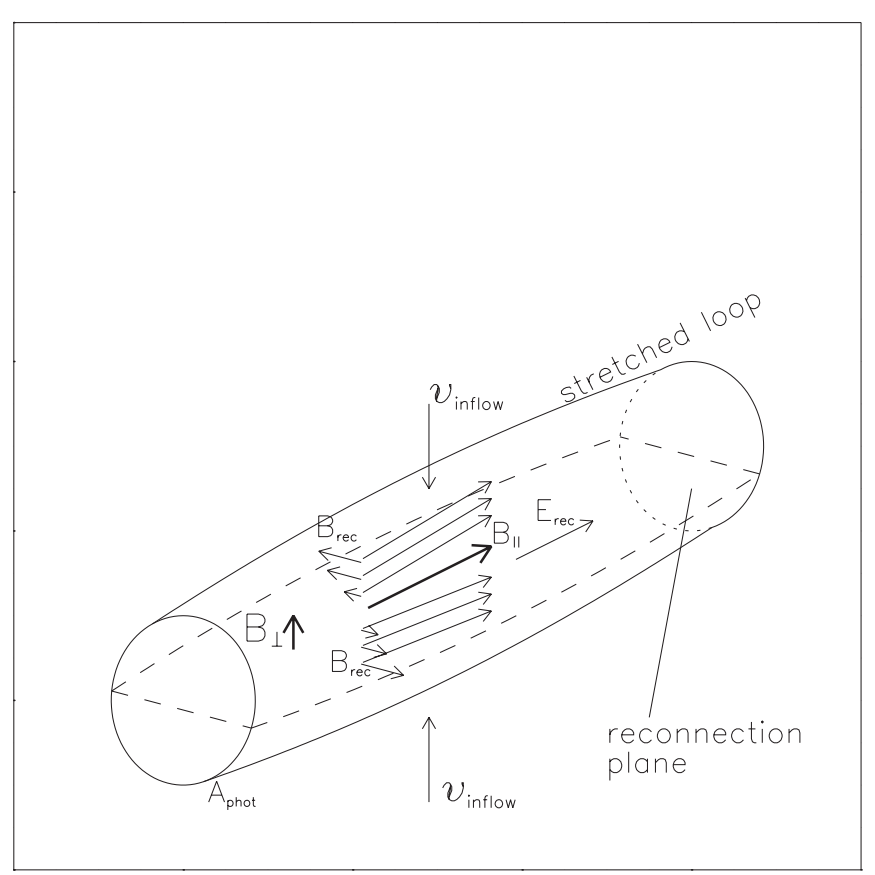

Figure 3. Stretched cylindroidal coronal loop. The lateral areas $A_{\text {phot }}$ correspond to the loop footpoints. The current sheet is formed at a reconnection plane parallel to the cylindroid's main axis. The magnetic field changes orientation in the half-cylindroids defined by the reconnection plane. The magnetic field projection perpendicular to the cylinder's axis forms the reconnecting component $B_{\text {rec }}$ while the projection parallel to the axis forms the parallel magnetic field component $B_{\|}$. The component $B_{\perp}$ is perpendicular to the current sheet plane.

These magnetic flux tubes, which form individual coronal loops, have a linear cross section smaller than 100-200 km (Cirtain et al. 2013; Chen et al. 2013). Our magnetogram data, binned to 1 .' 1 , cannot resolve these sub-resolution structures; i.e., we do not have any observational evidence on the value of the angle $\theta_{D}$. Given this limitation, in the present study, we assigned a value of the angle $\theta_{D}$ to each loop by picking from a uniform distribution between $8^{\circ}$ and $50^{\circ}$. The minimum value $8^{\circ}$ corresponds to the upper limit of the mean inclination of magnetic fields in simulations of MHD turbulence (Rappazzo et al. 2007) while the upper value includes (twice the) Parker angles of $40^{\circ}$ and $45^{\circ}$ derived from numerical simulations (Dahlburg et al. 2009; Galsgaard \& Nordlund 1996). Moreover, the distribution includes the typical value of $40^{\circ}$ found from energy considerations (Klimchuk 2006). We also attempted to calculate $\theta_{D}$ using the scaling law derived in Rappazzo et al. (2007). The results of the various calculations are discussed in detail in Section 6.

We assume that the shearing and twisting motions at the photospheric level are oriented such that the Poynting flux $S_{\text {phot }}$ is injected inward, i.e., into the sheet. For simplicity, we also assume that the current sheet is described by a Harristype geometry. In this geometry, two out of the three sheet's magnetic field components can be derived from the loop mean magnetic field $\bar{B}$ and $\theta_{D}$ : (1) the magnetic field component perpendicular to the loop axis and parallel to the current sheet plane is assumed to increase linearly with distance from the current sheet surface. Its maximum value $B_{\text {rec }}$, at the edges of the current sheet (see Figure 3), is given by $B_{\mathrm{rec}}=\bar{B} \sin \left(\theta_{D} / 2\right)$. (2) The magnetic field component parallel to the loop axis $B_{\|}$corresponds to the current sheet's guide field component. This component is assumed to be constant at each point inside 
the current sheet, and it is expressed as $B_{\|}=\bar{B} \cos \left(\theta_{D} / 2\right)$. The dimensionless parameter $\xi_{\|}=B_{\|} / B_{\text {rec }}$ is important in the current sheet literature (Efthymiopoulos et al. 2005; Litvinenko 2000). In the present modeling, it is given by $\xi_{\|}=\cot \left(\theta_{D} / 2\right)$. According to the above definitions, for $\theta_{D}=0$, the tangential discontinuity vanishes as $B_{\mathrm{rec}}=0$ and $B_{\|}=\bar{B}$. For $\theta_{D}=\pi$, we end up with an anti-parallel reconnection with $B_{\|}=0$ and $B_{\mathrm{rec}}=\bar{B}$. For the adopted range of $\theta_{D}$ between $8^{\circ}$ and $50^{\circ}$, we end up with $\xi_{\|}$between 14.3 and 2.15 , respectively. With the above settings, the injected Poynting flux $S_{\text {rec }}$ is expressed by the energy conservation equation as

$$
2 S_{\text {rec }} A_{\text {rec }}=A_{\text {phot }}\left(\left|S_{\text {foot1 }}+S_{\text {foot } 2}\right|\right) .
$$

In Equation (4), $A_{\text {rec }}$ is the current sheet area and the factor of two accounts for the fact that the Poynting flux is injected from both sides of the current sheet. Therefore, in Figure 3, $A_{\text {rec }}$ corresponds to the area of the dashed current sheet. $A_{\text {rec }}$ is calculated along each loop by integration, taking into account that the loop diameter normal to the loop's axis varies along the loop. $A_{\text {rec }}$ takes values in the range $10-760 \mathrm{Mm}^{2}$, with longer loops exhibiting larger values of $A_{\text {rec }}$.

The inflowing Poynting flux is directly related to the induced electric field $\boldsymbol{E}_{\text {rec }}$ that accelerates particles inside the current sheet. The electric field is given by

$$
\boldsymbol{E}_{\text {rec }}=-\frac{1}{c}\left(\boldsymbol{v}_{\text {inflow }} \times \boldsymbol{B}_{\text {rec }}\right),
$$

where $\boldsymbol{v}_{\text {inflow }}$ is the velocity of the plasma injected in the current sheet. The velocity $\boldsymbol{v}_{\text {inflow }}$ can be derived from the expression of the injected Poynting flux $S_{\text {rec }}$ via

$$
\boldsymbol{S}_{\mathrm{rec}}=\frac{c}{4 \pi}\left(\boldsymbol{v}_{\text {inflow }} \times \boldsymbol{B}_{\text {rec }}\right) \times \boldsymbol{B}_{\text {rec }} .
$$

Figure 4 presents the results of the above calculations. Figure 4(a) shows that the injected $S_{\text {rec }}$ values are in the range $10^{4}-2 \times 10^{9} \mathrm{erg} \mathrm{s}^{-1} \mathrm{~cm}^{-2}$. Furthermore, $S_{\text {rec }}$ decreases with increasing loop length because it is inversely proportional to $A_{\text {rec }}$. For short loops $(L<30 \mathrm{Mm})$, the mean injected Poynting flux is $S_{\text {rec }} \simeq 7.5 \times 10^{7} \mathrm{erg} \mathrm{s}^{-1} \mathrm{~cm}^{-2}$. For intermediate size loops $(30 \mathrm{Mm}<L<100 \mathrm{Mm})$, we find $S_{\text {rec }} \simeq 2.2 \times$ $10^{7} \mathrm{erg} \mathrm{s}^{-1} \mathrm{~cm}^{-2}$. While for long loops $(L>100 \mathrm{Mm}), S_{\mathrm{rec}} \simeq$ $7 \times 10^{6} \mathrm{erg} \mathrm{s}^{-1} \mathrm{~cm}^{-2}$. Here we assume that in an electron-proton plasma at thermal equilibrium, electrons will be accelerated practically without friction (due to Coulomb interactions with the protons), because the electric field applied to the plasma is much larger than the Dreicer electric field computed for standard coronal temperatures and plasma densities (Dreicer 1959; Benz 1993). In general, it is expected that electric fields appearing during solar-flare reconnection events are much larger than the Dreicer electric field (Martens \& Young 1990). An explicit computation of the Dreicer field values of our model is given at the end of this section.

As seen in Figure 4(b), 99\% of the electric field values are between 0.01 and $100 \mathrm{~V} \mathrm{~m}^{-1}$. The electric fields are also decreasing with increasing loop length. Moreover, $96 \%$ of the inflow velocity values are in the range $0.1-100 \mathrm{~km} \mathrm{~s}^{-1}$ (see Figure 4(c)) but there appears to be no correlation between $v_{\text {inflow }}$ and loop length.

The next important parameter to compute is the magnetic field component $B_{\perp}$ that is perpendicular to the plane of the current sheet (see Figure 3). Assuming consistency between the current
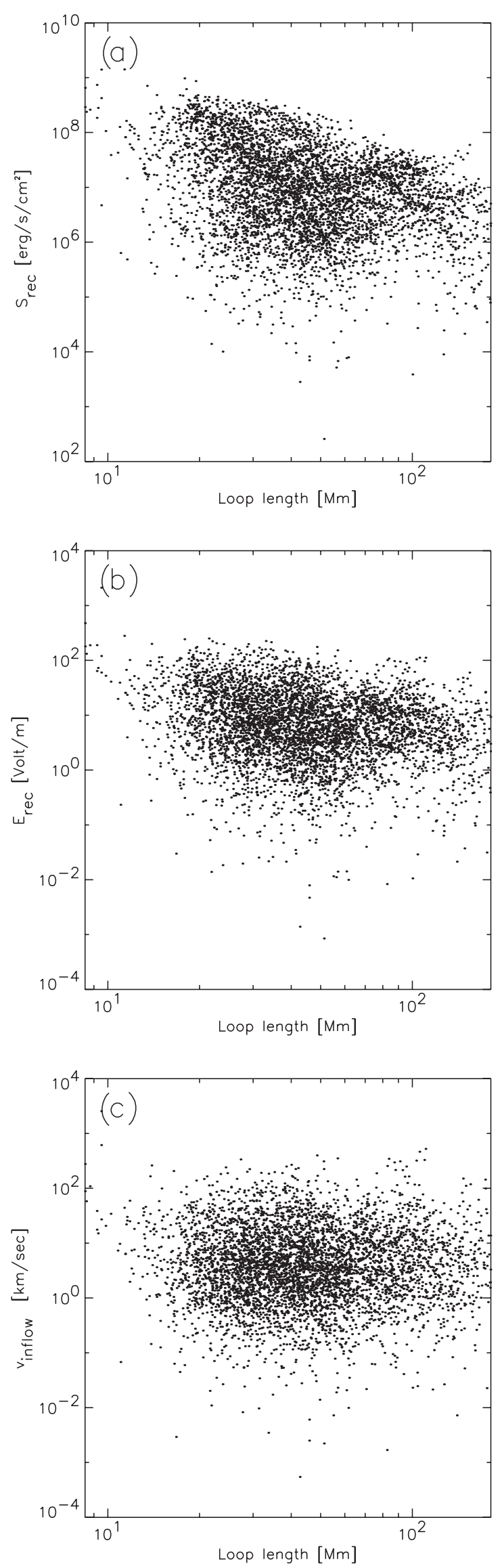

Figure 4. Scatter plots of (a) the injected Poynting flux, (b) the reconnecting electric field, and (c) the plasma velocity inflow inside each of our considered current sheets. 
derived from Ampére's law and the electric current produced by the accelerated protons (since they carry most of the particle energy), one finds (Eastwood 1972; Martens \& Young 1990; Litvinenko 1996)

$$
v_{A} B_{\perp}=\sqrt{2} v_{\text {inflow }} B_{\text {rec }}
$$

where $v_{A}$ is the Alfvén speed. The above equation is valid for $\xi_{\|}=0$. In the absence of a guide component, the reconnecting component $B_{\text {rec }}$ equals the average magnetic field $\bar{B}$ so that in Equation (7) the Alfvén speed can be expressed as $v_{A}=$ $B_{\text {rec }} / \sqrt{4 \pi m_{p} n_{e}}$. Equation (7) differs from the one found in Eastwood (1972), Martens \& Young (1990), or Litvinenko (1996) by a factor of $\sqrt{2}$. This numerical factor is introduced because we estimate the proton kinetic energy as being twice as large as in the above-mentioned works, for reasons explained in Section 5. For $\xi_{\|} \neq 0$, on the other hand, the reconnecting field component is not equal to the mean magnetic field. Therefore, $\bar{B}$ should replace $B_{\text {rec }}$ in the Alfvén speed expression through the definition $B_{\text {rec }}=\bar{B} \sin \left(\theta_{D} / 2\right)$. Inserting $\sin \left(\theta_{D} / 2\right)$, Equation (7) becomes

$$
v_{A} B_{\perp}=v_{\text {inflow }} B_{\text {rec }} \frac{\sqrt{2}}{\sin \frac{\theta_{D}}{2}} .
$$

We note that in the limit $\theta_{D}=\pi$ (anti-parallel reconnection), Equation (8) becomes identical to Equation (7).

In order to compute the Alfvén speed, we use the Rosner et al. (1978) scaling laws to determine the electron density for each loop assuming a maximum temperature of $T_{\max }=10^{6} \mathrm{~K}$. These scaling laws are valid in the strict sense for hydrostatic atmospheres. Their use in the present context will be discussed in Section 9. Because of the relatively low value of $T_{\max }$, we also have low electron densities in the range $2 \times 10^{8} \mathrm{~cm}^{-3}$ up to $6 \times 10^{9} \mathrm{~cm}^{-3}$.

Figure 5(a) shows the Alfvén speed for all our current sheets. The values of $v_{A}$ range from $10^{3}$ to $1.6 \times 10^{4} \mathrm{~km} \mathrm{~s}^{-1}$; these are expected values for the coronal plasma. From Equation (8), we compute $B_{\perp}$ for all the current sheets (Figure 5(b)), with $80 \%$ of the values in the range $0.01-1 \mathrm{G}$. The average $B_{\perp}$ is $0.3 \mathrm{G}$ and its standard deviation is $0.85 \mathrm{G}$. Moreover, for the dimensionless quantity $\xi_{\perp}=B_{\perp} / B_{\text {rec }}, 98 \%$ of the values are in the range of $10^{-5}$ to 1 .

Finally, we estimate the current sheet thickness as follows: according to Cassak et al. (2008), a current sheet becomes collisionless and susceptible to reconnection when its thickness $a$ becomes of the order of the ion gyroradius. However, when the guide magnetic field component is non-zero, instability leading to reconnection occurs when the current sheet thickness becomes of the order of the Hall scale $a=v_{s}(T) / \omega_{c i}$ (Cassak et al. 2007), where $v_{s}$ is the sound speed calculated for a plasma temperature $T=10^{6} \mathrm{~K}$ and $\omega_{c i}$ is the ion-cyclotron frequency. The current sheet thickness can also be expressed as $a=5.69 \times 10^{-8} v_{s} \bar{B}^{-1}\left(m_{p} / m_{e}\right)$. The resulting thickness turns out to be of the order of the electron gyroradius. Figure 5(c) shows that the computed thicknesses in our current sheet sample range from 0.01 to $1 \mathrm{~m}$, with the longer loops supporting thicker current sheets. Using the electron density $n_{e}$ and temperature $T=10^{6} \mathrm{~K}$, we also calculated the Dreicer electric field, which is given by the expression $E_{D}=6.06 \times 10^{-6} n_{e} / T \mathrm{~V} \mathrm{~m}^{-1}$ (Benz 1993). Our computed electric fields $E_{\mathrm{rec}}$ are larger than the Dreicer electric fields by factors ranging from 1 up to $10^{4}$ for $97 \%$ of the cases. We conclude that the assumption of collisionless acceleration of the particles in our modeled current sheets is consistent with the adopted plasma parameters.
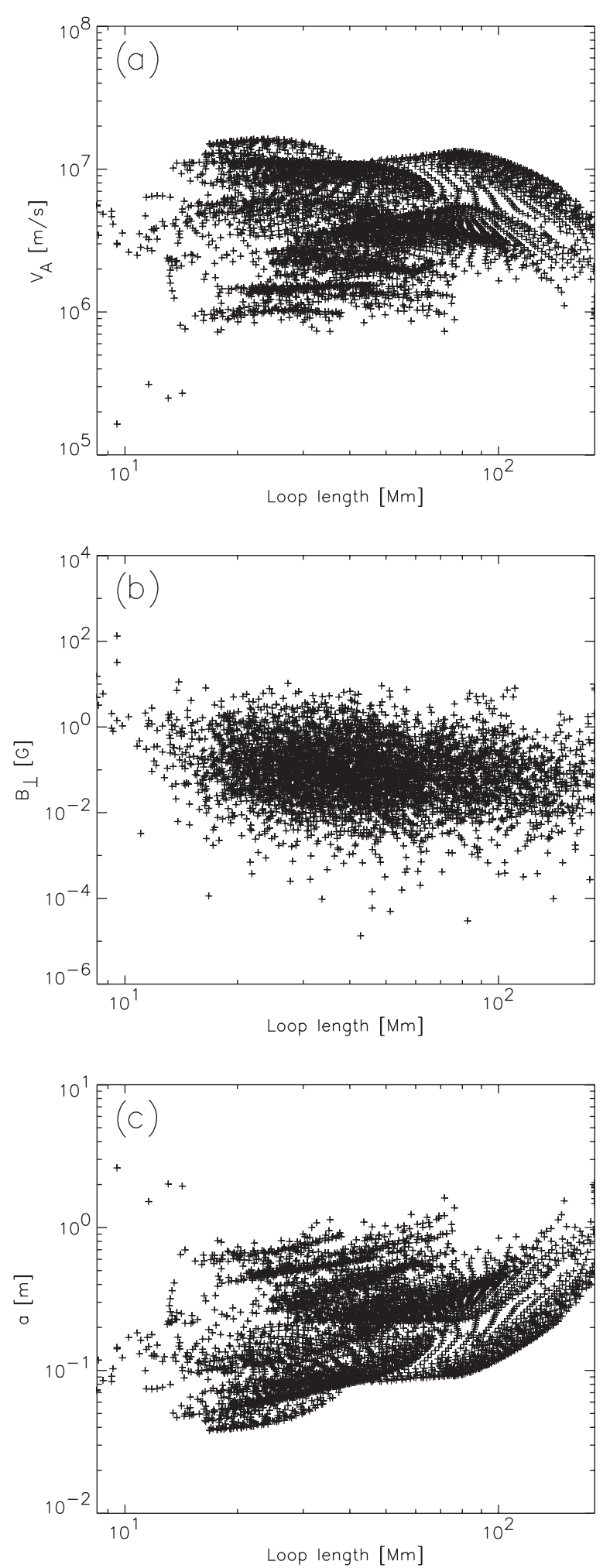

Figure 5. Scatter plots of (a) the Alfvén speed, (b) the perpendicular component $B_{\perp}$, and (c) the current sheet thickness, as a function of the loop length, for all current sheets considered.

\section{PARTICLE ACCELERATION}

In this section, we compute estimates of the kinetic-energy gain of electrons and protons accelerated through the loop current sheets considered in the previous section. In our approach, charged particles enter the current sheet with a velocity $v_{\text {inflow }}$ 
and are accelerated by the induced DC electric field $E_{\text {rec}}$. Inside the current sheet, particles follow a trajectory that depends on the initial velocity and strength of the electric and magnetic fields. Particles are ejected before they can travel along the total length of the current sheet, which in our case is equal to the loop length. Ejection is due to the Lorenz force created by the $B_{\perp}$ component (Speiser 1965). For current sheets with $\xi_{\|}>1$, particles will follow adiabatic orbits with a very small amount of chaos (Efthymiopoulos et al. 2005). In the present study, we did not examine the possibility of a particle interacting with more than one current sheet. Therefore, after being ejected from the current sheet, particles move along the magnetic field lines without any further acceleration. The particles' kinetic-energy gain is proportional to the electric field strength multiplied by the final acceleration length (along the electric field; see Figure 3). As the electric fields $E_{\text {rec }}$ are super-Dreicer, collisions are ignored. Therefore, to estimate the final kinetic energy, one needs to compute the orbit as long as the particle is under the influence of the current sheet electric and magnetic fields. Efthymiopoulos et al. (2005) derived an analytical expression for the kinetic-energy range $E_{k}$ of accelerated particles inside a Harris-type current sheet as a function of the initial particle energy $E_{k 0}$ and the current sheet's parameters (field strengths and thickness). This expression reads

$$
\begin{aligned}
E_{k j}= & E_{k 0}+\frac{E_{\mathrm{rec}}}{B_{\perp}^{2}}\left(e B_{\|} B_{\perp} a+m_{j} E_{\mathrm{rec}}\right. \\
& \left. \pm \sqrt{2 e m_{j} a B_{\|} B_{\perp} E_{\mathrm{rec}}+m_{j}^{2} E_{\mathrm{rec}}^{2}+2 m_{j} B_{\perp}^{2} E_{k 0}}\right) .
\end{aligned}
$$

$j$ represents the particle species (electrons or protons). The two values for the \pm sign define the energy range around a mean kinetic energy. In the following, we consider only the upper limit of the particles' kinetic-energy range (plus sign in Equation (9)). Furthermore, we consider that the initial particle energies obey a Maxwellian distribution at a temperature of $10^{6} \mathrm{~K}$.

In Efthymiopoulos et al. (2005), the analytical expression is more cumbersome than Equation (9). In this prior work, the expression includes explicitly $I_{2}$, an integral of the motion of particle orbits. $I_{2}$ results from the translational symmetry of the Harris-type sheet geometry along $\boldsymbol{E}_{\text {rec }}$ (see Figure 3; Efthymiopoulos et al. 2005; Litvinenko \& Somov 1993). In Equation (9), we assume for simplicity that $I_{2}=0$. However, even with this assumption, Equation (9) gives a good estimate of the final kinetic energy of the particles. The first two terms in Equation (9)

$$
E_{k e}=E_{k 0}+\frac{e E_{\mathrm{rec}} B_{\|} a}{B_{\perp}}
$$

are sufficient to describe the electrons' average final kinetic energy (Litvinenko 2000). For the protons, the kinetic energy is well described by the expression (Litvinenko 2000; Efthymiopoulos et al. 2005)

$$
E_{k p}=2 m_{p} c^{2}\left(\frac{E_{\mathrm{rec}}}{B_{\perp}}\right)^{2} .
$$

The factor of two arises because in Equation (9), the $m_{p} E_{\text {rec }}$ term occurs twice and results in the $\sqrt{2}$ factor in Equations (7) and (8). The initial particle kinetic energy, corresponding to a Maxwellian kinetic energy for the selected temperature of the order of $0.04 \mathrm{keV}$, is, on average, 40 and 800 times smaller than the final kinetic energy of the electrons and protons, respectively, after the acceleration process. The final electron kinetic energies are of the order of $0.1-8 \mathrm{keV}$ while the final proton kinetic energies are in the range $0.3-470 \mathrm{keV}$ (Figure 6).

Figure 6(a) shows the kinetic-energy distribution of electrons while Figure 6(b) shows the kinetic-energy distribution of protons. The largest kinetic energies are found for loops with lengths ranging between 15 and $30 \mathrm{Mm}$. In Figures 6(a) and (b), the kinetic-energy scatter plots for electrons and protons have a similar shape. This is because the kinetic energy of electrons depends on the Alfvén velocity while the kinetic energy of protons depends on the square of the Alfvén velocity. This dependence on the Alfvén velocity, which is implicit in Equations (10) and (11), comes from the definition of $B_{\perp}$ in Equation (7). This dependence becomes explicit after the following calculation. Combining Equations (5), (6), (8), (10), and (11), as well as the definitions of the thickness $a$ and the $B_{\|}$ component, we obtain the dependence of the sum of electron and proton kinetic-energy gain on the initial parameters:

$$
\begin{aligned}
E_{k} & =\frac{e E_{\mathrm{rec}} B_{\|} a}{B_{\perp}}+2 m_{p} c^{2}\left(\frac{E_{\mathrm{rec}}}{B_{\perp}}\right)^{2} \\
& =\frac{e}{c \sqrt{2}} \bar{B} \cos \frac{\theta_{D}}{2} a v_{A} \sin \frac{\theta_{D}}{2}+2 m_{p} c^{2}\left(\frac{v_{A} \sin \frac{\theta_{D}}{2}}{c \sqrt{2}}\right)^{2} \\
& \Rightarrow E_{k}=\frac{e}{c 2 \sqrt{2}} c_{1} v_{s}(T) v_{A} \sin \theta_{D}+m_{p} v_{A}^{2} \sin ^{2} \frac{\theta_{D}}{2} .
\end{aligned}
$$

Equation (12) holds for $0<\theta_{D}<\pi$ since, for $\theta_{D}=0$, we have no current sheet while for $\theta_{D}=\pi$ the reconnection is antiparallel $\left(\xi_{\|}=0\right)$ and one should use Equation (9). Moreover, we omitted the initial kinetic energy $E_{k 0}$ from Equation (12). In Equation (12), the constant $c_{1}=5.8 \times 10^{-8}\left(m_{p} / m_{e}\right)$ originates from the expression for the thickness $a$ (Cassak et al. 2007), introduced in Section 4. Equation (12) shows that the final kinetic energy $E_{k}$ of the accelerated particles depends on the Alfvén velocity, the plasma density, and the discontinuity angle $\theta_{D}$. The sound velocity $v_{s}(T)$ is introduced through the thickness $a$ of the current sheet. The kinetic energy $E_{k}$ is implicitly related to $S_{\text {rec }}$, as seen in Figure 6(c), where we observe that $S_{\text {rec }}$ and $E_{k}$ are well correlated. Moreover, the electron kinetic energy as a function of the mean magnetic field at the loop footpoints is well fit by the power law $E_{k e} \propto \bar{B}_{\text {foot }}^{0.65}$. This means that for a different $\mathrm{AR}$ with stronger photospheric magnetic fields, reaching, for example, $5000 \mathrm{G}$, we expect that electron kinetic energies can reach up to $10 \mathrm{keV}$. Another important aspect is that $E_{k}$ is independent of the surface area $A_{\text {rec }}$ of the current sheet. This means that the initial selection of the current sheet to cover the entire surface of the loop cross section does not influence the resulting kinetic energies. This allowed us to choose, for each loop, a single current sheet with a simple geometry to describe the particles' acceleration.

In addition, the acceleration length $z_{\max }$ of the particles is an important parameter in our model. The acceleration length is the distance along the electric field covered by the particles and it is defined via the expression $E_{k}=e E_{\mathrm{rec}} z_{\max }$ for both particle species. $z_{\max }$ must obviously be smaller than the current sheet length or, equivalently, the coronal loop length. We found that for electrons, the ratio $z_{\max } / L$ is between $10^{-7}$ and $10^{-3}$ in $97 \%$ of cases, while for protons it is between $10^{-6}$ and 0.01 in $90 \%$ of cases. For protons, the average value of $z_{\max } / L$ is $10^{-3}$ with a standard deviation of 0.1 . A short acceleration length relative to the loop length reduces the electric current intensity which, in turn, reduces the induced magnetic fields generated by the electric current of the accelerated particles (Martens \& Young 1990; Litvinenko 1996). 

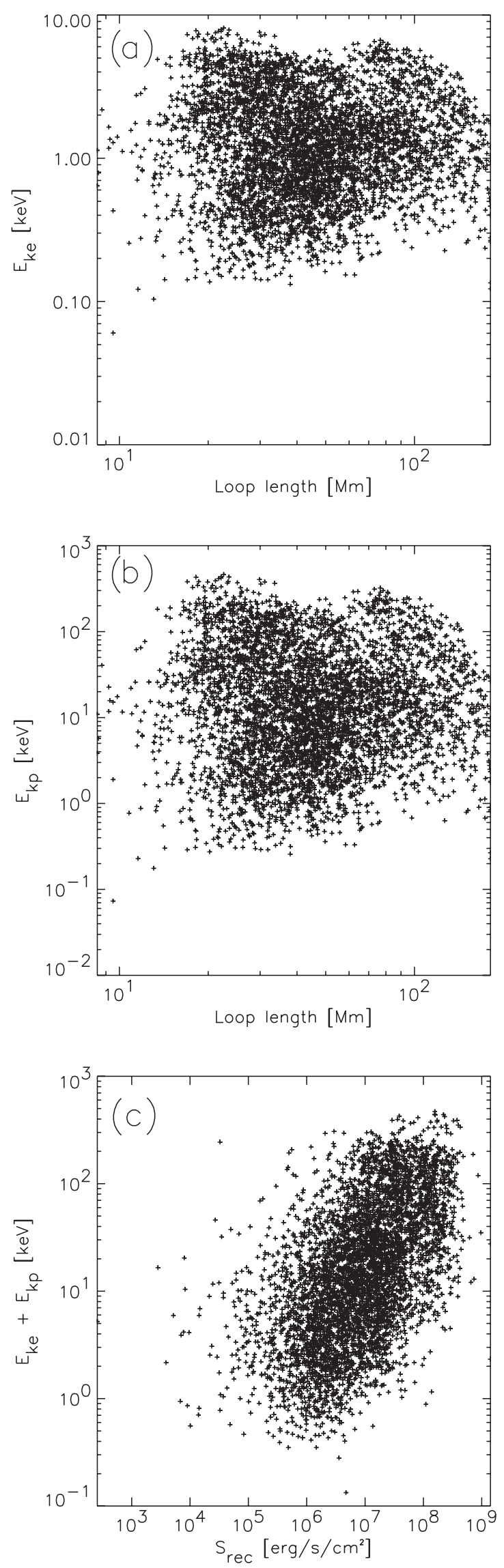

Figure 6. Scatter plots of the final kinetic energy for electrons (panel (a)) and protons (panel (b)). Panel (c) shows the total kinetic energy of electrons and protons as a function of the Poynting flux entering the current sheet.
We performed test particle simulations for the acceleration of electrons and protons similar to the ones presented in Gontikakis et al. (2007) and Anastasiadis et al. (2008). In these simulations, single particles, initially with a random $10^{6} \mathrm{~K}$ thermal velocity, enter a Harris-type current sheet with given initial parameters ( $\left.a, B_{\|}, B_{\perp}, E_{\mathrm{rec}}, B_{\mathrm{rec}}\right)$. Solving the equations of motion, a particle's orbit is traced until the particle leaves the current sheet at a half-width distance from the inversion surface. Running simulations for all 5000 current sheets used in this study is unrealistic, given time constraints. Therefore, we selected 10 representative values of the electric field $E_{\text {rec }}$, reconnecting parallel and perpendicular magnetic components $\left(B_{0}, B_{\perp}, B_{\|}\right)$, and current sheet thicknesse $a$ from our calculated distributions. The resulting kinetic energies are in agreement with Equation (9). All 1000 electrons used in each simulation are accelerated to kinetic energies of the order inferred by Equation (10). However, most protons cross the current sheet with no energy gain and only roughly $10 \%$ of the protons are accelerated. The accelerated protons are the ones that have an initial velocity with a particular orientation relative to the current sheet. The fact that only a fraction of protons are accelerated was also found by Gontikakis et al. (2007).

To recapitulate, we use observations of photospheric magnetic fields and inferences of the photospheric horizontal velocities to estimate the particles' kinetic energies. From the energies, we calculate the photospheric Poynting flux $S_{\text {phot }}$ (Equation (2)). From a potential magnetic field extrapolation, we select 5000 closed loops, for each of which we calculate the mean magnetic field $\bar{B}$. From this and by virtue of the assumptions described above, we calculate the parameters $B_{\text {rec }}, B_{\|}, v_{\text {inflow }}, E_{\text {rec }}, a$, and $B_{\perp}$ that are necessary and sufficient to calculate the kinetic energies of accelerated particles.

\section{LOOP HEATING DUE TO ACCELERATED PARTICLES}

We compute the heating of coronal loops assuming that heat is produced solely by the ensuing thermalization of the accelerated particles. We assume that both electrons and protons participate in this process, since both particles' energy is released once they hit the dense chromospheric layer. Protons with energies ranging between $100 \mathrm{keV}$ and $5 \mathrm{MeV}$ can produce chromospheric evaporation, thus participating in coronal heating (Emslie et al. 1996). In our simulation, only $10 \%$ of the protons' kinetic energies are above $100 \mathrm{keV}$. Nevertheless, we assume that even protons with kinetic energies less than $100 \mathrm{keV}$ participate in the loop heating.

The thermalization of the accelerated particles is a complex process that we do not model in detail. Instead, we use the following phenomenological expression for the heating rate:

$$
Q=\frac{\left(E_{k e}+f E_{k p}\right) n_{e}}{t_{\mathrm{rec}}} \frac{V_{\mathrm{rec}}}{V_{\mathrm{loop}}} .
$$

The heating rate $Q$ of a loop with a pre-nanoflare electron density $n_{e}$ corresponds to the thermalization of current-sheetaccelerated electrons and protons to kinetic energies $E_{k e}$ and $E_{k p}$, respectively. A neutral, fully ionized plasma $\left(n_{e}=n_{p}\right)$ is assumed. Here, $V_{\text {rec }}=2 v_{\text {inflow }} t_{\text {rec }} A_{\text {rec }}$ is the plasma volume injected inside the current sheets of total surface $A_{\text {rec }}$ during the reconnection time $t_{\text {rec }}$. We define the reconnection time as the duration of the reconnection event. The fraction $V_{\text {rec }} / V_{\text {loop }}$ indicates that the heating produced inside the current sheets is redistributed to the loop volume $V_{\text {loop }}$. The efficiency factor $f=0.1$ means that only $10 \%$ of the protons are accelerated, 

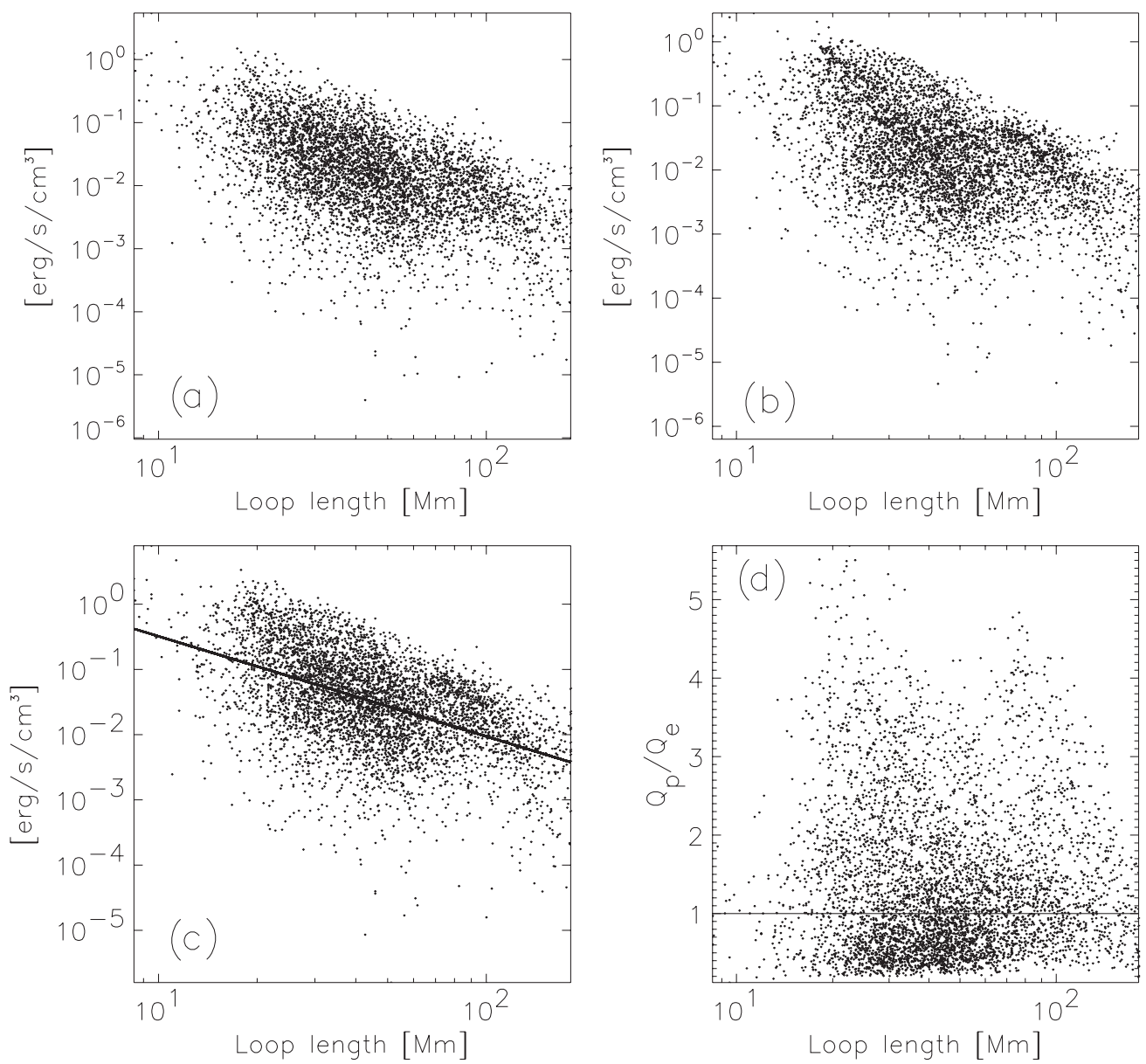

Figure 7. Heating rates produced by electrons (panel (a)), protons (panel (b)), and by both particle types (panel (c)) as a function of loop length. Panel (d) shows the ratio of proton heating to electron heating as a function of loop length. Panel (c) also shows a linear fit to the log of the heating rate distribution.

as found by the test particle simulations presented at the end of Section 5.

In subsequent calculations, we use Equations (4), (5), and (12) and assume that the loop volume is $V_{\text {loop }}=C_{4} L^{-p}$, where $C_{4}=50.1, L$ is the loop length, and $p=1.85$ as found by the fit to the calculated loop volumes in Figure 2(c). The heating $Q$ is expressed (in $\mathrm{erg} \mathrm{s}^{-1} \mathrm{~cm}^{-3}$ ) as

$$
\begin{aligned}
Q= & \frac{4 \pi}{c}\left(\frac{e}{c 2 \sqrt{2}} c_{1} \sin \theta_{D} v_{s}(T) v_{A}\right. \\
& \left.+f m_{p} v_{A}^{2} \sin ^{2} \frac{\theta_{D}}{2}\right) \frac{n_{e} \bar{S}_{\mathrm{foot}} A_{\mathrm{phot}}}{B_{\mathrm{rec}}^{2} C_{4} L^{p}} .
\end{aligned}
$$

Here, $\bar{S}_{\text {foot }} A_{\text {phot }}$ is the average photospheric Poynting flux times the footpoint cross section. In Equation (14), we can see that the heating rate $Q$ does not depend on $t_{\text {rec }}$ and that the electron and proton terms have different dependencies on $v_{A}$. We can also express $Q$ as a function of $L$ if we replace the electron density $n_{e}$ by the expression given in Rosner et al. (1978). The individual heating rates due to electrons and protons have a power-law dependence on $L$ but slightly different exponents:

$$
\begin{gathered}
Q_{e} \propto \bar{S}_{\text {foot }} \frac{\cot \frac{\theta_{D}}{2} T v_{s}(T)}{\bar{B} L^{\frac{1+2 p}{2}}} \\
Q_{p} \propto \bar{S}_{\text {foot }} \frac{1}{L^{p}} .
\end{gathered}
$$

Figure 7 shows the heating rates due to electrons (Figure 7(a)), protons (Figure 7(b)), and both types of particles (Figure 7(c)) as a function of loop length. Despite the larger scatter, a similar power-law dependence of the heating produced by each type of particle, shown in Equation (15), is also visible in the scatter plots of Figures 7(a)-(c). The heating-flux ratio $Q_{p} / Q_{e}$ seen in Figure 7(d) takes values in the range $0.1-5$ with $50 \%$ of the values larger than 1 . The heating rate function from the two particle species (Figure 7(c)) is in the range of $10^{-4}$ to $1 \mathrm{erg} \mathrm{s}^{-1} \mathrm{~cm}^{-3}$. We also show a linear fit to the logarithm of the values in the scatter plot. The calculated power-law index is $c_{2}=-1.5$. This index differs from $p=1.85$, the exponent of $L$ in Equation (15). The reason is that $\bar{S}_{\text {foot }}$, which appears in Equation (15), also has a weak dependence on $L$ with a positive power-law index $a=0.35$. This dependence influences the resulting fit. In Mandrini et al. (2000), the heating rate deduced from loops observed in X-rays with the soft X-ray telescope on Yohkoh has a power-law relation with the loop length. Exponents are in the interval $[-4.5,-1]$ and the most probable value is $\simeq-2$. Therefore, the exponent value of -1.5 presently derived is fairly consistent with observations. In Figure 8, we plot the ratio of the total heating $Q_{p+e}=Q_{p}+Q_{e}$ due to electrons and protons over the value of the Poynting flux function $h_{\text {Poynt }}$ injected inside the loops (plotted in Figure 2). We observe that $95 \%$ of the values $Q_{p+e} / h_{\text {Poynt }}$ are in the range $9-50$ with an average of 21 .

Thus, the heating rate found in our model considering particle acceleration overestimates the one recovered from the Poynting 


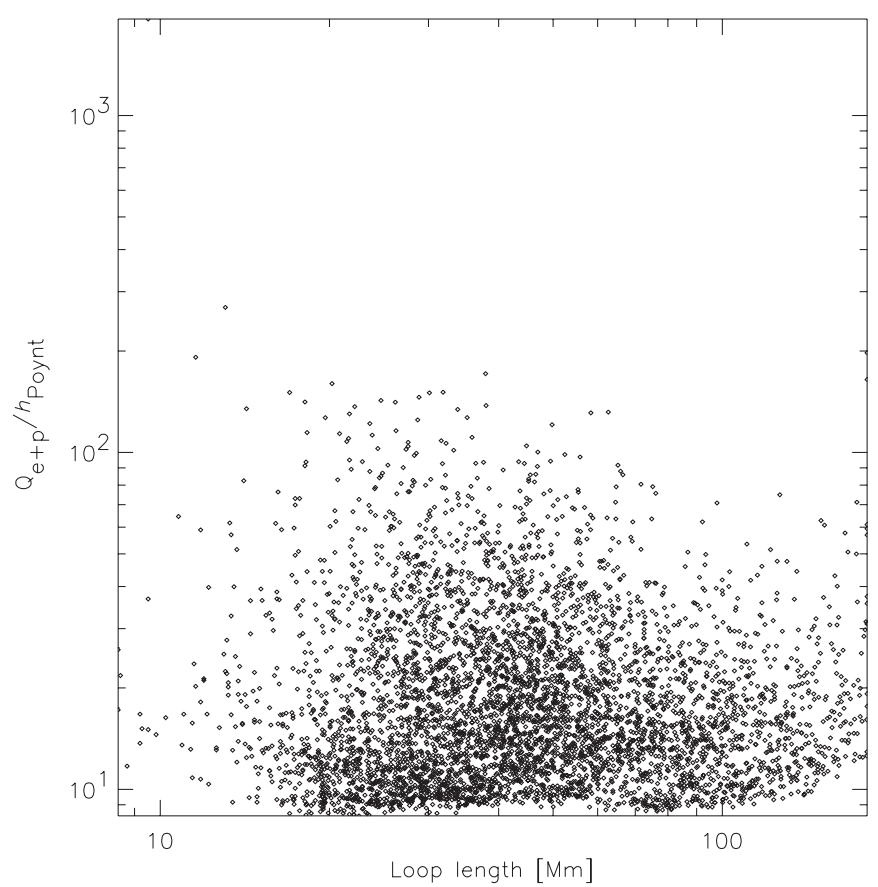

Figure 8. Ratio of the heating rates due to particles over $h_{\text {Poynt }}$, the energy rate corresponding to the Poynting flux.

flux by an average factor of $\simeq 20$. This appears at first as a large inconsistency. However, as pointed out by Rosdahl \& Galsgaard (2010) and Birn \& Priest (2007, p. 287), this inconsistency should be regarded as a consequence of the inherent lack of self-consistency in all methods estimating particle acceleration via the trajectories of test particles. In fact, in MHD simulations of coronal heating (Hendrix et al. 1996; Galsgaard \& Nordlund 1996; Rappazzo et al. 2007), one finds $Q_{p+e} / h_{\text {Poynt }} \simeq 1$. However, in such simulations, the balance is restored partly due to an additional feedback mechanism caused by the ongoing twisting and relaxation of magnetic fields. On the other hand, the effects of magnetic reconnection on the acceleration of particles cannot be captured by such simulations. At any rate, our present results show that the thermalization of the loops is indeed possible, at least as shown by order of magnitude estimates, via the conversion of magnetic energy to particles' kinetic energy during magnetic reconnection.

One more feature to note is that the possible lifetime of a single current sheet could be of the order of the Alfvén crossing time, which is equal to the fraction of the current sheet length over the Alfvén speed (Klimchuk 2006). In our model, this time ranges from 1-135 s. On the other hand, hydrodynamic simulations of nanoflares consider a storm of many nanoflares with a duration of 50-500 s (Klimchuk 2006). Let us note that the ratio $V_{\text {rec }} / V_{\text {loop }}$ is less than 1 for $95 \%$ of our current sheets, if we set their lifetimes equal to the Alfvén crossing time. This means that for $5 \%$ of the loops, reconnection would practically end because of the lack of sufficient plasma inflow. For the other cases, only a fraction of the total number of particles stored in each loop was able to cross the current sheet during the reconnection event. Moreover, the time needed for an individual particle to cross the current sheet is much smaller than any of the above timescales. For electrons, the particle acceleration time is in the range $10^{-6}$ to $0.01 \mathrm{~s}$, while for protons it is in the range $10^{-4}$ to $1 \mathrm{~s}$. In $98 \%$ of the cases, the acceleration times of protons turn out to be smaller than one tenth of the corresponding current sheet lifetime. This justifies our assumption that the particles interact with a quasi-stationary environment during the acceleration phase.

An additional remark is that our computed accelerated particles' kinetic energies and heating functions are still valid if, instead of a monolithic current sheet per loop, we assumed a large number of current sheets. However, we require that these sheets are formed by the same discontinuity angle $\theta_{D}$ and the same magnetic field components $B_{\text {rec }}, B_{\perp}, B_{\|}$. Furthermore, the sheets must have the same thickness and they must be longer than the corresponding acceleration length $z_{\max }$. Moreover, we only consider particles interacting with a single current sheet during their travel inside the loop. Thus, Equation (13) describes the heating per current sheet, which is proportional to a fraction $S_{\text {foot }} A_{\text {phot }}$ of the heating rate supplied. In Section 8 , we will calculate the hydrodynamic response of the studied loops under the effect of a number of current sheets producing the same collective heating effect as that described in Equation (13).

To summarize the results of Sections 5 and 6, Figure 9 shows some model parameters as a function of $\theta_{D}$. These parameters were presented in previous figures as a function of the loop length. The inflow velocity $v_{\text {infow }}$ (Figure $9(a)$ ) has higher values for a lower $\theta_{D}$. In Figure 9(b), the kinetic energies of electrons decrease with increasing $\theta_{D}$ according to Equation (12), where the first term in the last sum depends on $\sin \theta_{D}$. On the other hand, $Q_{e}$ is higher for lower $\theta_{D}$ (Figure $9(\mathrm{c})$ ), as expected from Equation (15). Finally, in Figure 9(d), one can see that for higher $\theta_{D}, Q_{e+p} / h_{\text {Poynt }}$ tends asymptotically to a lower limit of $\simeq 8$.

We also calculated $\theta_{D}$ according to a scaling law given in Equation (10) in Rappazzo et al. (2007). The main parameter in this equation is the ratio of photospheric velocity $v_{p h}$ over the Alfvénic velocity $v_{A}$. For our data, the derived $\theta_{D}$ were in all cases less than $4^{\circ}$. The small $\theta_{D}$ values are due to the low ratio of photospheric to Alfvénic velocities in our model. Alfvén velocities are high due to the low $n_{e}$ calculated using the scaling law of Rosner et al. (1978) with a relatively low temperature of $10^{6} \mathrm{~K}$. As shown in Figure 9, such small $\theta_{D}$ values correspond to even larger $Q_{e+p} / h_{\text {Poynt }}$ ratios. However, as pointed out in Rappazzo et al. (2007), Equation (10) should be regarded only as a lower limit of the Parker angle, as it does not take into account the current sheet formation. On the other hand, much higher values of $\theta_{D}$ are predicted by Hendrix et al. (1996) and Galsgaard \& Nordlund (1996).

\section{THE AVERAGE THICK-TARGET X-RAY SPECTRUM FROM NANOFLARES}

In this section, we attempt to model the expected form of the thick-target spectrum produced by the electrons in the AR loops. We first calculate the distribution of the accelerated electrons' kinetic energy. The total number of electrons, $N_{e}$, produced by each loop is given by the product of the loop electron density $n_{e}$ (given by Rosner et al. 1978) multiplied by the plasma volume $V_{\text {rec }}$ entering the current sheet during the reconnection time $t_{\text {rec}}$. Therefore, $N_{e}=2 v_{\text {inflow }} t_{\text {rec }} n_{e} A_{\text {rec }}$. We assume that the kinetic-energy distribution in each loop lies in the energy range $\Delta E=\left[E_{\mathrm{kin}(\min )}, E_{\mathrm{kin}(\max )}\right]$, where $E_{\mathrm{kin}(\min )}$ and $E_{\mathrm{kin}(\max )}$ are the energy limits calculated by Equation (9). The amplitude of the kinetic-energy distribution of each loop is equal to $g=N_{e} / \Delta E$ and is assumed to be constant inside $\Delta E$. To compute the kinetic-energy distribution of all selected loops, we divide the energy $E$ from $0.1 \mathrm{keV}$ to $7 \mathrm{keV}$ in 100 energy bins of $\delta E=0.07 \mathrm{keV}$. For each energy bin, at a given energy $E$, we take the sum of the individual loop distribution amplitudes $g_{j}$ 

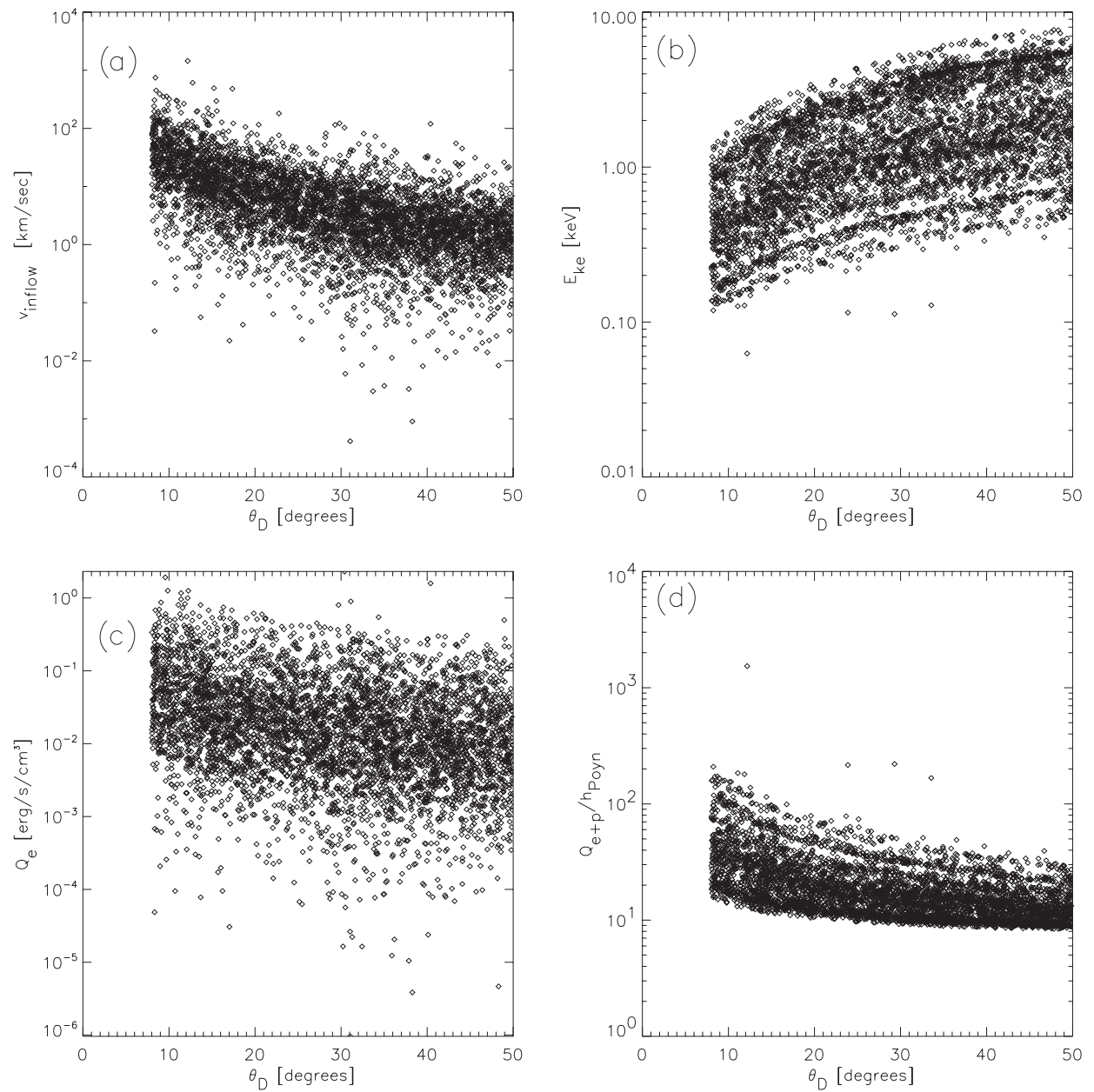

Figure 9. Some key quantities of our model as a function of $\theta_{D}$. Panel (a) shows the $v_{\text {inflow }}$ velocity, panel (b) shows the final kinetic energy of electrons, panel (c) shows the heating due to the accelerated electrons, and panel (d) shows the ratio of heating due to accelerated electrons and protons over the heating corresponding to the supplied Poynting flux. In all panels, the horizontal axis shows $\theta_{D}$ values for each loop.
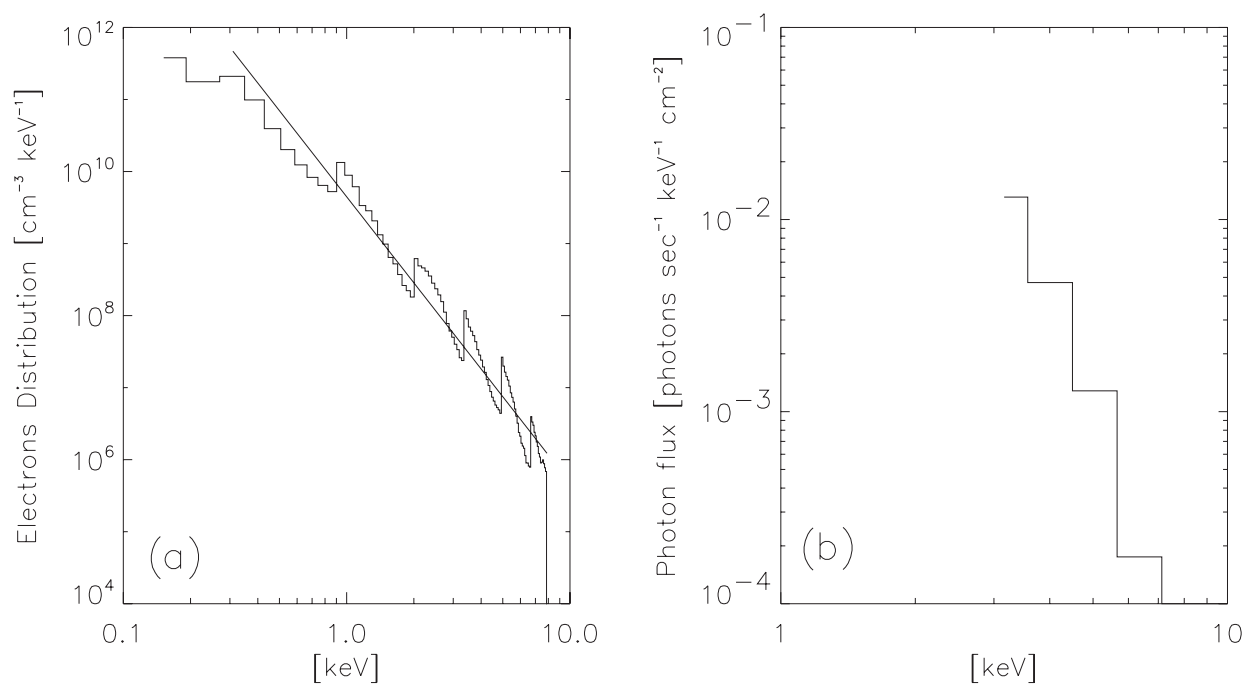

Figure 10. Average kinetic-energy distribution from the 5000 selected loops (panel (a)) and the corresponding X-ray spectrum (panel (b)) calculated according to the thick-target approximation.

for which $\left(E_{\operatorname{kin}(\min ) j}<E<E_{\operatorname{kin}(\max ) j}\right)$. Also, for each energy bin, we take the sum of the volumes $V_{\text {loop } j}$ of the corresponding loops. Finally, the average kinetic-energy distribution $F(E) d E$ is calculated at each energy bin $i$ as $F\left(E_{i}\right)=\Sigma g_{j} / \Sigma V_{\text {loop } j}$ in units of electrons $\mathrm{cm}^{-3} \mathrm{keV}^{-1}$. Note that this distribution assumes that nanoflares from all loops are triggered simultaneously, which is one more simplification. Our derived AR kineticenergy distribution is shown in Figure 10(a). We performed a power-law fit of the form $F=G E^{b}$ and found an exponent $b \simeq-4$ and a proportionality factor $G=3.3 \times 10^{9} \mathrm{~cm}^{-3} \mathrm{keV}^{-1}$. 
We used the derived fit parameters to compute the X-ray spectrum assuming the thick-target approximation (Brown 1971) using the Fortran code developed by Holman (2001). We assumed that the area of the radiating source function equals the sum of the loop footpoint areas $\left(A=7 \times 10^{19} \mathrm{~cm}^{2}\right)$. The resulting X-ray spectrum is seen in Figure $10(\mathrm{~b})$. The X-ray spectrum is divided by the number of loops (5000) assuming that on average only one nanoflare is active at a time. Therefore, the computed X-ray flux represents a lower limit of the nanoflare emission. The computed X-ray flux per loop has a maximum of $10^{-2}$ photons $\mathrm{s}^{-1} \mathrm{keV}^{-1} \mathrm{~cm}^{-2}$ at $3 \mathrm{keV}$ and falls to $10^{-4}$ photons $\mathrm{s}^{-1} \mathrm{keV}^{-1} \mathrm{~cm}^{-2}$ at $7 \mathrm{keV}$. Our derived X-ray spectrum exhibits a narrow energy range because of the narrow energy range of the electron kinetic energies. For this reason, a comparison of the spectrum shape with low solar activity X-ray observed spectra, such as the RHESSI data presented in McTiernan (2009) or the SphinX data presented in Miceli et al. (2012), was not attempted. However, our computed X-ray flux is of the same order of magnitude as the upper limits measured in the quiet Sun with RHESSI (Hannah et al. 2011, p. 278, their Figure 12, left panel).

\section{LOOP HYDRODYNAMIC RESPONSE TO NANOFLARES}

In this section, we employ the heating rates computed via Equation (13) as an input to hydrodynamic loop simulations. This allows us to determine the thermal response (differential emission measure, DEM) to the heating resulting from our model. As the DEM can also be deduced by observations, a comparison between simulated and observed DEMs is a standard test for coronal models.

We assume that each loop is heated due to the activation of several current sheets of sub-telescopic sizes, which inject beams of accelerated particles into the loops. Each current sheet is activated for a duration of the order of the Alfvén crossing time along the loop, but the nanoflare cascade duration is different and subject to parameterization.

We also assume that after the particles (electrons and protons) are accelerated somewhere along the loop, they exit the current sheet and deposit their kinetic energy, via Coulomb collisions, to the lower and denser parts of the loop (i.e., the thick-target model of electron beams; e.g., Brown 1971). However, the general characteristics (e.g., maximum temperature and density) of loops submitted to thermal (i.e., direct) and non-thermal (i.e., particle) heating do not substantially differ for the same total energy release (e.g., Warren \& Antiochos 2004). Differences could arise in the ultra-hot plasma $\left(>5 \times 10^{6} \mathrm{~K}\right)$, during the early stages of impulsive heating (e.g., Klimchuk et al. 2008) or in the mass flows from the loop footpoints, which are however not considered here. Instead, particle heating is treated here as thermal heating. Finally, the particle beams follow the magnetic field lines directly connected to their individual current sheet, whereas the macroscopic loop is heated due to the collective effect of all individual current sheets.

In order to study the loop plasma response to the calculated heating rates, we use the EBTEL model of Klimchuk et al. (2008). EBTEL is a zero-dimensional model, i.e., it considers spatially averaged properties and uses analytical approximations to solve the time-dependent hydrodynamic equations. It was found to be in good agreement with simulations using far more complex, yet computationally expensive, one-dimensional models. By definition, the heating is assumed to be spatially uniform in EBTEL. Allowing for different scenarios of the

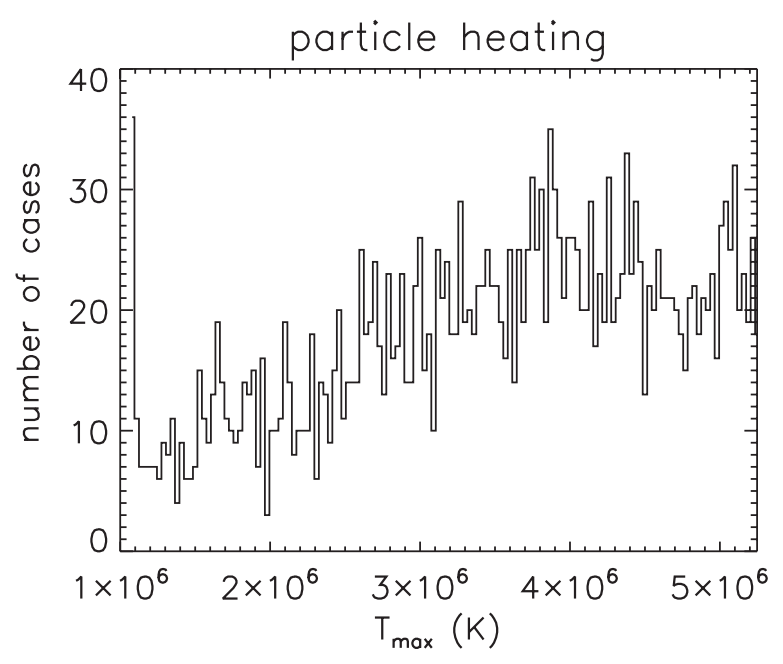

Figure 11. Histogram of temperatures achieved at the maximum of each loop time-averaged DEM distribution. This calculation is performed for $t_{\text {heat }}=100 \mathrm{~s}$.

spatial localization of the heating leaves distinct signatures only during the early stages of the hydrodynamic evolution, when the loop is at very high temperatures (e.g., Patsourakos \& Klimchuk 2005). For a given heating profile, loop length, and initial temperature and density conditions, EBTEL calculates at any instance the coronal temperature and density as well as the transition region (footpoint) and coronal DEM. The application of our model to the observations of NOAA 09114 described in the previous paragraphs supplies each loop hydrodynamic simulation with the length and the corresponding heating rate. Given that the model is zero dimensional and analytical, it can calculate numerous solutions in reasonable time.

Our hydrodynamic calculation starts with initial conditions determined according to the scaling law of Rosner et al. (1978). We presume a coronal temperature of $1 \mathrm{MK}$. (However, note that this scaling law is derived in the hydrostatic limit. For the use of this approximation, see Section 9.) Then, at the initial timestep $(t=0 \mathrm{~s})$, each loop was impulsively heated according to Equation (13). Each loop is heated impulsively by the corresponding heating rate given in Figure 7 . The heating took the form of a step function with a duration of $t_{\text {heat }}$. Numerical simulations (Georgoulis et al. 1998) predict a wide distribution for the duration of heating events in nanoflares. Therefore, $t_{\text {heat }}$ is a free parameter for our model, and we run simulations with values of $t_{\text {heat }}=15,50,100,250$, and $500 \mathrm{~s}$ for all the loops. We also considered the case of $t_{\text {heat }}$ equal to the Alfvén crossing time for each loop. Since $t_{\text {heat }}$ is in general longer than the Alfvén crossing time, this computation corresponds to a storm of nanoflares when different fragments of the loop current sheet are activated at different times. The employed $t_{\text {heat }}$ values are comparable to the duration of small-scale impulsive energy release events found in MHD simulations of coronal heating. For each loop, the corresponding simulations lasted $5000 \mathrm{~s}$ to allow us to follow both the heating and cooling of the plasma.

In Figure 11, we plot the histogram of the temperatures of the peak of the temporally averaged DEM of each loop computed for $t_{\text {heat }}=100 \mathrm{~s}$. The vertical axis represents the number of loops per temperature bin. We can see that the particle heating creates an almost uniform distribution of temperatures. The peak of the distribution at $10^{6} \mathrm{~K}$ corresponds to loops that are not significantly influenced by heating; these loops maintain their initial temperature. For $t_{\text {heat }}=50 \mathrm{~s}$, the temperature histogram is very similar to the one shown in Figure 11 . For $t_{\text {heat }}=500 \mathrm{~s}$, 


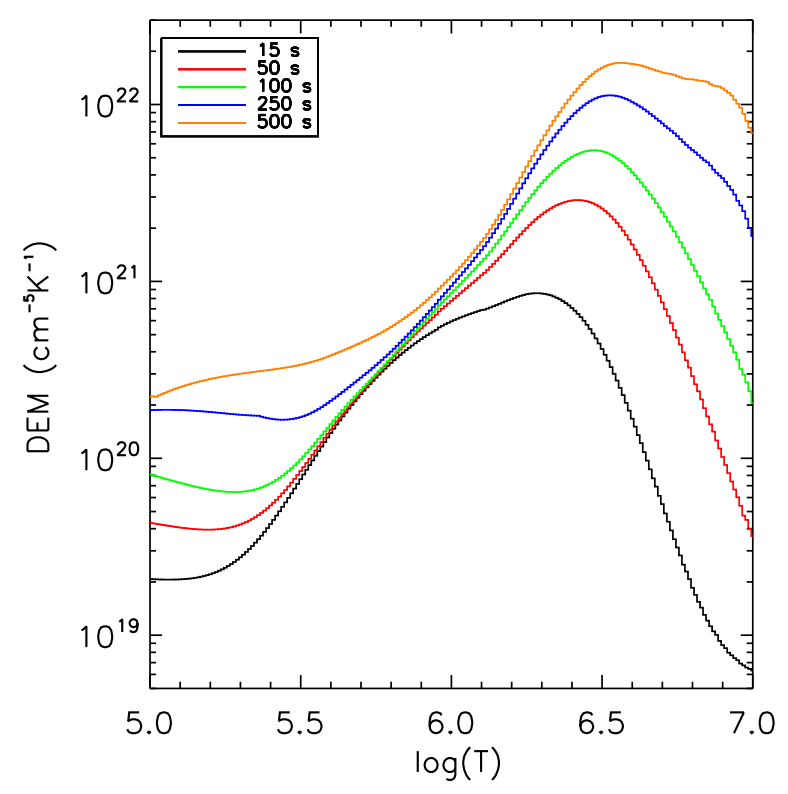

Figure 12. Differential emission measure calculated assuming that the heating is caused by particle acceleration. The plot is shown for different $t_{\text {heat }}$ values.

(A color version of this figure is available in the online journal.)

the distribution exhibits a plateau in the range of $2 \mathrm{MK}$ up to $7 \mathrm{MK}$, while for $t_{\text {heat }}=15 \mathrm{~s}$, the histogram shows a higher probability of temperatures lower than $2 \mathrm{MK}$.

In Figure 12, we plot the time-averaged DEMs for all the considered loops in NOAA AR 09114. The plotted DEMs correspond to both the coronal and footpoint parts of the modeled loops. This is legitimate because we deal with averages over the entire AR that would obviously include contributions from both the coronal and footpoint regions. The latter are known to supply most of the low-temperature emission at around $1 \mathrm{MK}$, particularly in AR cores.

DEMs provide the amount of plasma present at each temperature bin and therefore offer an idea of the thermal distribution of the region or feature in question. Several remarks are now in order from Figure 12. The deduced DEMs have maximum values of $9 \times 10^{20}, 2 \times 10^{21}, 4 \times 10^{21}, 10^{22}$, and $1.5 \times 10^{22} \mathrm{~cm}^{-5} \mathrm{~K}^{-1}$ for $t_{\text {heat }}$ of $15,50,100,250$, and $500 \mathrm{~s}$, respectively. The temperature of the maximum DEM value rises for higher heating durations; the maximum $\log (T)$ is in the range of 6.4-6.6.

Observationally deduced DEMs (e.g., Landi \& Landini 1997; O'Dwyer et al. 2011: their Figures 4 and 14, respectively) exhibit a broad peak from $\log (T)=6-6.5$ at a value of a few times $10^{21} \mathrm{~cm}^{-5} \mathrm{~K}^{-1}$. At both limits of this plateau, DEMs drop off rapidly. Therefore, the DEMs from our model can reproduce the features of observed AR DEMs. Once again, we emphasize that in the framework of our proof-of-concept calculations, we are not aiming to reproduce any particular observational details.

\section{DISCUSSION}

Since a rather large number of assumptions and/or approximations were introduced in our modeling of nanoflare heating presented in the previous sections, we summarize here the main limitations and conditions of validity of our model. We also discuss possible future extensions.

\subsection{Model Limitations}

An important limitation of our model is that it does not describe the initial transformation of the photospheric Poynting flux into magnetic free energy. When a certain critical value of the Parker angle is reached, this magnetic free energy presumably is released back into the plasma, causing heating. Here, we simply assumed that all loops reach the critical point (Parker angle) at which the Poynting flux is transformed into particle acceleration.

It should be noted that reconnection is a complex non-steady phenomenon (Loureiro et al. 2007; Samtaney et al. 2009). The presence of a guide magnetic field component has an important influence that is not yet fully understood (Yamada et al. 2010; Birn \& Priest 2007). Another important approximation is the use of a Harris-type analytical geometry to study the orbits of accelerated particles. Such an approach does not take into account either the perturbations caused by the accelerated particles onto the fields or the more complex structures of the magnetic field topology that we expect to be formed at the reconnection sites.

The scaling laws of Rosner et al. (1978) were used in order to compute initial conditions for our nanoflare simulations, as well as for the calculation of kinetic energies and heating rates in the previous sections. However, these formulae are valid in the strict sense for hydrostatic atmospheres, while in reality all parameters in our calculations should exhibit some time dependence. One may remark, nevertheless, that the characteristic timescale of evolution of the atmosphere is determined by the time $t_{\text {evap }}$ needed by the chromospheric evaporation flows to fill the loops with dense and hot plasma. According to some large flare simulations (Yokoyama \& Shibata 1998), chromospheric evaporation flows propagate at $20-30 \%$ of the speed of sound, at a temperature of $\simeq 4 \times 10^{6} \mathrm{~K}$. We find $t_{\text {evap }}=L /\left(0.4 v_{s}\right)$ in the range $100-500 \mathrm{~s}$ for $80 \%$ of our cases. The resulting timescales are of the same order as the highest $t_{\text {heat }}$ values used in our calculations. At any rate, Yokoyama \& Shibata (1998) argue that a chromospheric evaporation flow should not influence the reconnection rate at a flare's X-point. In view of the above, and since $t_{\text {evap }}$ is typically larger or at most equal to $t_{\text {heat }}$, we conclude that the use of the Rosner et al. (1978) scaling laws in our heating computations is an allowable approximation. A more accurate computation would require a proper application of a chromospheric evaporation particle heating rate feedback model. This is proposed for future work.

\subsection{Comparison with Other Models}

Some words are necessary to explain our choice of a hydrodynamic model. There are basically two approaches to studying coronal loop heating based on (1) three-dimensional MHD (e.g., Gudiksen \& Nordlund 2005; Peter et al. 2006; Dahlburg et al. 2012; Bingert \& Peter 2011) or (2) onedimensional and zero-dimensional hydrodynamic simulations. Three-dimensional MHD simulations supply a physics-based heating function (e.g., ohmic heating at intense current sheets formed at the interfaces of braided magnetic elements). However, three-dimensional MHD simulations lack the spatial resolution available in one-dimensional hydrodynamic simulations. A high resolution, on the other hand, is crucial for an accurate description of the plasma thermodynamic response to a given heating. Nevertheless, the heating functions selected in one-dimensional hydrodynamic loop simulations are ad hoc; i.e., they can be chosen arbitrarily. Finally, hydrodynamic descriptions are far less computationally expensive than threedimensional MHD simulations, and thus are more appropriate for extensive studies of thousands of loops. 
A future improvement concerns the geometry of our model. Replacing the Harris current sheet with a more realistic geometry would allow us to calculate test particle orbits for a range of parameters pertinent to solar ARs, over a large number of coronal loops. Of course, the ultimate improvement would be to simulate the feedback of the plasma response due to the chromospheric evaporation on the acceleration of the particles.

\section{CONCLUSIONS}

In the present study, we provide a set of calculations for nanoflare heating in coronal loops. We base our calculations on a composite model in which the heating term used for hydrodynamic simulations of nanoflares derives from considering particle acceleration in reconnecting current sheets. Our main steps and conclusions are the following.

1. Our calculations utilize observational data: (1) the general structure of the magnetic field is deduced by means of a current-free (potential) magnetic field extrapolation of an observed AR's (NOAA AR 9114) magnetogram. (2) We selected 5000 closed magnetic field lines derived from the extrapolation to represent coronal loops. We study the nanoflares in these loops. (3) The Poynting flux is supplied in current sheets, one for each coronal loop, produced by photospheric motions at the loop footpoints. The Poynting flux is calculated using the measured magnetic fields and estimated values for the inductive velocities at the photospheric level.

2. In our current sheets, reconnection always occurs because we assume that the discontinuity in the magnetic field configuration has reached a critical mis-alignment angle. The mis-alignment angle, $\theta_{D}$ (twice the adopted Parker angle), varies randomly from loop to loop in a uniform distribution from $8^{\circ}$ to $50^{\circ}$. In this model, we compute the physical conditions in the current sheets. The induced electric field $E_{\text {rec }}$ is in the range of $0.01-100 \mathrm{~V} \mathrm{~m}^{-1}$ and is larger than the Dreicer electric field that favors the direct acceleration of particles. The plasma inflow velocity $v_{\text {inflow }}$ is in the range of $0.1-100 \mathrm{~km} \mathrm{~s}^{-1}$.

3. The Poynting flux supplied by the photospheric motion is entirely transformed into kinetic energy of the particles, accelerated in the reconnecting current sheets. The final kinetic energies of electrons and protons are calculated using an analytic formula derived in test particle studies (Efthymiopoulos et al. 2005; Litvinenko 1996). The electron kinetic-energy gain is up to $8 \mathrm{keV}$, while for protons the gain is in the range $0.3-470 \mathrm{keV}$.

4. We consider the process of particles' acceleration as the unique source of plasma heating. This assumption is supported by the fact that at least in large flares, electron acceleration corresponds to $50 \%$ of the released energy (Birn \& Priest 2007). We use a simple phenomenological expression (Equation (13)) to compute the heating rate produced by accelerated electrons $\left(Q_{e}\right)$ and protons $\left(Q_{p}\right)$. The produced heating rates are in the range of $10^{-4}$ to $1 \mathrm{erg} \mathrm{s}^{-1} \mathrm{~cm}^{-3}$ while the ratio $Q_{p} / Q_{e}$ takes values in the range $0.1-5$ and is higher than 1 in $50 \%$ of the cases.

The power law of the computed heating rate as a function of the loop length, derived both via a fit to the calculated data and via an analytical derivation, has an exponent of $\simeq-1.5$. This value falls within the constraints derived from observations (Mandrini et al. 2000). Moreover, we found a linear dependence of the heating functions on the Poynting flux at the footpoints and a trigonometric dependence on the angle $\theta_{D}$.

5. We computed the form of X-ray spectra generated by the accelerated electrons from all loops, using the "thick-target" approach. The derived spectrum has a peak intensity of $10^{-2}$ photons $\mathrm{s}^{-1} \mathrm{keV}^{-1} \mathrm{~cm}^{-2}$ at $3 \mathrm{keV}$ and decreases with a power-law shape and an exponent equal to $\simeq-4$. This result is in agreement with upper limits derived from observations (Hannah et al. 2011).

6. Finally, we performed hydrodynamic simulations using the zero-dimensional EBTEL code to compute the characteristic atmospheres of our loops. The constraints of the simulations are the derived heating rates and the loop length while the heating event duration is kept as a free parameter. The deduced DEMs have maximum values in the range $9 \times 10^{20}-1.5 \times 10^{22} \mathrm{~cm}^{-5} \mathrm{~K}^{-1}$ for temperatures from 6.4 to 6.6 in $\log (T)$. These derived values are in agreement with DEMs derived from observations (Landi \& Landini 1997; O’Dwyer et al. 2011).

7. We discuss the various limitations of our model and propose a number of possible future extensions as well as comparisons with other models in the literature.

We thank the anonymous referee for helpful and constructive comments. This research was supported in part by the European Union (European Social Fund ESF) and in part by the Greek Operational Program "Education and Lifelong Learning" of the National Strategic Reference Framework (NSRF)—Research Funding Program: Thales, "Hellenic National Network for Space Weather Research"-MIS 377274. S.P. acknowledges support from an FP7 Marie Curie Grant (FP7-PEOPLE2010-RG/268288). C.G. acknowledges support from program 200/790 of the research committee of the Academy of Athens.

\section{REFERENCES}

Alissandrakis, C. E. 1981, A\&A, 100, 197

Anastasiadis, A., Gontikakis, C., \& Efthymiopoulos, C. 2008, SoPh, 253, 199

Benz, A. O. 1993, Plasma Astrophysics (Dordrecht: Kluwer)

Bingert, S., \& Peter, H. 2011, A\&A, 530, 112

Birn, J., \& Priest, E. (ed.) 2007, Reconnection of Magnetic Fields (Cambridge: Cambridge Univ. Press)

Brown, J. C. 1971, SoPh, 18, 489

Cassak, P. A., Drake, J. F., \& Shay, M. A. 2007, PhPl, 14, 054502

Cassak, P. A., Mullan, D. J., \& Shay, M. A. 2008, ApJ, 676, 69

Chen, B., Bastian, T. S., White, S. M., et al. 2013, ApJ, 763, 21

Cirtain, J. W., Golub, L., Winebarger, A. R., et al. 2013, Natur, 493, 501

Dahlburg, R. B., Einaudi, G., Rappazzo, A. F., \& Velli, M. 2012, A\&A, 544, 20

Dahlburg, R. B., Klimchuk, J. A., \& Antiochos, S. K. 2005, ApJ, 622, 1191

Dahlburg, R. B., Liu, J.-H., Klimchuk, J. A., \& Nigro, G. 2009, ApJ, 704, 1059 Dreicer, H. 1959, PhRv, 115, 238

Eastwood, J. W. 1972, P\&SS, 20, 1555

Efthymiopoulos, C., Gontikakis, C., \& Anastasiadis, A. 2005, A\&A, 443, 663

Emslie, A. G., Hénoux, J.-C., Mariska, J. T., \& Newton, E. K. 1996, ApJ, 470,131

Galsgaard, K., \& Nordlund, Å. 1996, JGR, 101, 13445

Georgoulis, M. K. 2005, ApJ, 629, 69

Georgoulis, M. K., \& LaBonte, B. J. 2006, ApJ, 636, 475

Georgoulis, M. K., Velli, M., \& Einaudi, G. 1998, ApJ, 497, 957

Gontikakis, C., Anastasiadis, A., \& Efthymiopoulos, C. 2007, MNRAS, 378, 1019

Gontikakis, C., Contopoulos, I., \& Dara, H. C. 2008, A\&A, 489, 441

Gudiksen, B. V., \& Nordlund, А̊ 2005, ApJ, 623, 600

Handy, B. N., Bruner, M. E., Tarbell, T. D., et al. 1998, SoPh, 183, 29

Hannah, I. G., Hudson, H. S., Battaglia, M., et al. 2011, SSRv, 159, 263

Harra, L. K., Archontis, V., Pedram, E., et al. 2012, SoPh, 278, 47

Hendrix, D. L., Van Hoven, G., Mikic, Z., \& Schnack, D. D. 1996, ApJ, 470,1192 
Hesse, M., Schindler, K., \& Kuznetsova, M. 1999, PhPl, 6, 1781

Holman, G. D. 2001, http://hesperia.gsfc.nasa.gov/hessi/modelware Klimchuk, J. A. 2006, SoPh, 234, 41

Klimchuk, J. A., Patsourakos, S., \& Cargill, P. J. 2008, ApJ, 682, 1351

Landi, E., \& Landini, M. 1997, A\&A, 327, 1230

Lemen, J. R., Title, A. M., Akin, D. J., et al. 2012, SoPh, 275, 17

Litvinenko, Y. E. 1996, ApJ, 462, 997

Litvinenko, Y. E. 2000, SoPh, 194, 327

Litvinenko, Y. E., \& Somov, B. 1993, SoPh, 146, 127

Loureiro, N. F., Schekochihin, A. A., \& Cowley, S. C. 2007, PhPl, 14, 100703

Mandrini, C. H., Demoulin, P., \& Klimchuk, J. A. 2000, ApJ, 530, 999

Martens, P. C. H., \& Young, A. 1990, ApJS, 73, 333

McTiernan, J. M. 2009, ApJ, 697, 94

Metcalf, T. R., Leka, K. D., Barnes, G., et al. 2006, SoPh, 237, 267

Miceli, M., Reale, F., Gburek, S., et al. 2012, A\&A, 544, 139

Mickey, D. L., Canfield, R. C., Labonte, B. J., et al. 1996, SoPh, 168, 229
O’Dwyer, B., Del Zanna, G., Mason, H. E., et al. 2011, A\&A, 525, 137

Parker, E. 1972, ApJ, 174, 499

Parker, E. 1988, ApJ, 330, 474

Patsourakos, S., \& Klimchuk, J. A. 2005, ApJ, 628, 1023

Patsourakos, S., Klimchuk, J. A., \& MacNeice, P. J. 2004, ApJ, 603, 322

Peter, H., Gudiksen, B. V., \& Nordlund, Å. 2006, ApJ, 638, 1086

Rappazzo, A. F., Velli, M., Einaudi, G., \& Dahlburg, R. B. 2007, ApJ, 657,47

Rosdahl, K. J., \& Galsgaard, K. 2010, A\&A, 511, 73

Rosner, R., Tucker, W. H., \& Vaiana, G. S. 1978, ApJ, 220, 643

Samtaney, R., Louleiro, N. F., Uzdensky, D. A., Schekochihin, A. A., \& Cowley, S. C. 2009, PhRvL, 103, 105004

Speiser, T. W. 1965, JGR, 70, 4219

Warren, H. P., \& Antiochos, S. K. 2004, ApJ, 611, 49

Yamada, M., Cruskud, R., \& Li, H. 2010, RvMP, 82, 603

Yokoyama, T., \& Shibata, K. 1998, ApJ, 494, 113 